\title{
KAPITALISTISCHE KRISE UND ARBEITSLOSIGKEIT IN DER BUNDESREPUBLIK
}

Zum Verhältnis von Überakkumulation von Kapital und Überproduktion von Waren, struktureller und zyklischer Arbeitslosigkeit in der aktuellen Krise 1974/75

\author{
Jürgen Hoffmann, Willi Semmler
}

\section{Einleitung}

Die Frage nach den ökonomischen Ursachen der Arbeitslosigkeit in der Bundesrepublik kann und darf nicht nur an der Entwicklung eines Zyklus festgemacht werden, sondern muß auf dem Hintergrund der historischen Entwicklung des Kapitalverhältnisses, der Entwicklung der Widersprüche in der Kapitalakkumulation und des Verhältnisses der Klassen zueinander, beantwortet werden. Wird dagegen nur das zyklische Auf und Ab betrachtet, können die historischen Kräfte hinter der Bewegung der kapitalistischen Gesellschaft kaum noch ausgemacht werden: schließlich gab es in Westdeutschland zyklische Krisen ohne die Entwicklung einer massiven Arbeitslosigkeit! Die aktuell hohe Arbeitslosigkeit gerät dann zum Betriebsunfall oder zur bewußt von Unternehmern zum Zwecke der Disziplinierung der Arbeiter produzierten massenhaften Freisetzung von Arbeitern. Um mit Werner Meißner zu sprechen: „wilde Investitionsstreiks“ bzw. ,spontane Kapitalniederlegungen" sind die Ursache der zyklischen Krise. (1)

Nicht nur, daß einer solchen Analyse die scheinbare Lösung der Widersprüche in der kapitalistischen Gesellschaft mittels Investitionskontrollen leicht fällt (2); eine solche Analyse verschleiert gerade moralisierend die Funktion der Krise und die Ansatzpunkte sozialistischer Politik: Daß sich nämlich in der Krise die Akkumulationsbedürfnisse des Kapitals und die Lebensbedürfnisse der Arbeiter unvereinbar gegenüber stehen; daß dieser dem Kapitalverhältnis immanente Antagonismus in der Unvereinbarkeit von Vollbeschäftigung, hinreichendem Reproduktionsniveau und kapitalistischer Form des Wachstums in der Krise aufbricht; daß die Herrschaft des Kapitals - wenn auch über Phasen der Prosperität verdeckt - Krisen notwendig hervorbringt. An den materiellen Erfahrungen der Arbeiter kann auf Basis solcher Theorien nicht mehr angeknüpft werden.

1) Vgl. Werner Meißner, Stabilisierungspolitik in der Bundesrepublik Deutschland, in: WSI-Forum, Stabilisierungspolitik, Köln 1974, WSI-Studie Nr. 27, S. 63

2) Dabei ist eine Sache, Investitionskontrollen im Rahmen einer Taktik des Klassenkampfes zu fordern und eine andere, kapitalistisches Wachstum über Investitionsplanung ",rationaler" gestalten zu wollen. Es soll hier also nicht gegen eine notwendige Übergangsforderung der Arbeiterklasse, sondern gegen die sozialdemokratische Illusion, durch Steuerung der privaten Investitionen den Kapitalismus krisenfreier machen zu können, polemisiert werden. 
Um diesen Gegensatz in der aktuellen Krise und die daraus ableitbaren Probleme sozialistischer Politik in den Gewerkschaften in ihren konkreten Dimensionen darzustellen, wollen wir zunächst das Verhältnis von Überakkumulation und Überproduktion im kapitalistischen Krisenzyklus klären. Wir müssen davon ausgehen, daß sich in der Folge der Marxschen Theorie zwei Hauptstränge marxistischer Krisentheorien herausgebildet haben, die die oben angeschnittenen Fragen zum Verhältnis von Kapital und Arbeit in der Krise auch politisch unterschiedlich beantworten:

a) Die Unterkonsumtions- oder Überproduktionstheorien führen aus der behaupteten unmittelbaren Identität von Überakkumulation von Kapital mit der Überproduktion von Waren die Krise auf die beschränkte Konsumtionsfähigkeit der Massen zurück. Die politischen Forderungen, die aus dieser theoretischen Basis abgeleitet werden, sind dann auch die nach der Steigerung der Massenkaufkraft, um die Realisierung des brachliegenden Warenkapitals und die Ausnutzung der Produktionskapazitäten zu ermöglichen. Demnach sind also die Akkumulationsbedürfnisse des Kapitals und die Lebensbedürfnisse der Arbeiter durchaus miteinander vereinbar. Denn eine solche Realisierungsmöglichkeit würde ja den in den Waren vergegenständlichten Wert (und Mehrwert) zu den Kapitalen zurückfließen lassen, während die Konsumtion der Arbeiter gehoben werden könnte. Die Profite und die Profitraten stiegen wieder, die Akkumulation würde wiederbelebt werden. (An diesem Punkt setzen auch linkskeynesianische Theorien an, wie sie von den Gewerkschaften vertreten werden: Steigerung der "effektiven Nachfrage " durch Steigerung der Massenkaufkraft). Ein gesellschaftlicher Gegensatz von Lohnarbeit und Kapital (als Herrschaftsverhältnis) kann hier also gar nicht materiell begründet werden; es wird darum in diesen Theorien nicht an den materiellen Erfahrungen dieses Gegensatzes angeknüpft, sondern - wenn überhaupt - an ungerechten Verteilungsverhältnissen, Unternehmerwillkür und allgemeinen gesellschaftlichen Benachteiligungen der Arbeiter. Reformismus und/ oder Aktionismus sind notwendige politische Ausdrücke dieser Theorien.

b) Dagegen beziehen die Überakkumulationstheorien eine Position, die bei dem Gegensatz von Lohnarbeit und Kapital als ökonomisch vermittelten Herrschaftsverhältnis ansetzt und die Gesetzmäßigkeiten der kapitalistischen Produktionsweise als materielle Erfahrungsbasis nimmt, von der ausgehend der Gegensatz politisch zu wenden ist. Nicht nur ungerechte Verteilungsverhältnisse und mangelnde Konsumtionskraft, sondern das Kapital als Subjekt der gesellschaftlichen Bewegung und als Schranke seiner selbst werden Zentrum der Analyse und Basis politischer Folgerungen. Höhere Löhne, so wird gegen die Unterkonsumtionstheoretiker gewandt, ist immer eine zentrale Forderung, die die Reproduktion der Arbeiter gewährleisten soll. Denn es kann nicht Aufgabe der Arbeiter sein, die Prosperität des Kapitals zu gewährleisten durch den Verzicht auf die eigene Reproduktion. Zugleich drückt sich darin, daß diese Forderung in Widerspruch steht zur Akkumulation des Kapitals und damit zur materiellen Sicherung des Arbeitsplatzes der antagonistische Gegensatz von Kapital und Lohnarbeit aus, der im Kapitalismus nicht aufzuheben ist, sondern zur Existenzbedingung des 
Kapitals gehört. Insofern diese Theorie also auf der Erfahrung des Antagonismus aufbaut, weist sie auf eine revolu tionäre Perspektive hin.

Allerdings setzt eine solch entwickelte Erfahrung nicht nur das Manifestwerden eines solchen Antagonismus, sondern auch den entwickelten Kampf der Arbeiter gegen das Kapital voraus. Zunächst sind die Arbeiter ja immer nicht nur von ihren konkreten Tätigkeiten her fraktioniert (während sich in der oben angesprochenen Erfahrung gerade das Schicksal der Lohnarbeit äußert), sondern auch durch die Zugehörigkeit zu einzelnen Kapitalen, mit deren Schicksal sie existentiell verbunden sind. So sind - gerade nach langjährigen Prosperitätsphasen wie z. B. in Westdeutschland, die ja einherging mit relativer Prosperität der Arbeiter, sozialpartnerschaftlichen Vorstellungen etc. - die unmittelbaren Anschauungen aus der Sicht des Einzelkapitals auch in der Erfahrung der Arbeiter (als Belegschaften eines Betriebes) dominant: Nicht das Kapital wird von den Arbeitern als Schranke ihrer Reproduktion erfahren, sondern die mangelnde Nachfrage nach den Produkten ,,ihres“ “ Betriebes. (In dieser Weise knüpfen die Unterkonsumtionstheorien nicht nur an den verkehrten Vorstellungen des Einzelkapitals an, sondern bestätigen in fataler Weise auch die sozialpartnerschaftlichen Illusionen von Teilen der Arbeiterschaft.

Aus der Sicht des Einzelkapitals (und auch des einzelnen Arbeiters) muß sich die Krise so als Überproduktion von Waren darstellen. Wir werden daher im folgenden versuchen, den Stellenwert der „Überproduktion von Waren“ im Verlauf der Überakkumulation darzustellen. Dazu werden wir zunächst noch einmal die wichtigsten Resultate der Untersuchung der langfristigen Trends der Kapitalverwertung in Westdeutschland zusammenfassen und auf die Entwicklung in den 70er Jahren beziehen, um dann am Beispiel des letzten Zyklus das Verhältnis von Überakkumulation und Überproduktion entwickeln zu können. Auf dieser Basis können dann die zyklischen und die langfristigen Ursachen der Herausbildung einer industriellen Reservearmee dargestellt werden und es können später die gewerkschaftliche Politik kritisiert und die aktuellen Bedingungen und Aufgaben sozialistischer Gewerkschaftsarbeit herausgearbeitet werden.

\section{ZUR ENTWICKLUNG DER AKKUMULATIONSBEDINGUNGEN DES KAPITALS IN DEN 7OER JAHREN}

In PROKLA 16 und 17/18 wurde versucht, die historische Entwicklung des Kapitalverhältnisses in Westdeutschland und den Zusammenhang von Kapitalverwertungskrise und Inflation darzustellen. ( 2 a) Dort wurde ausgegangen vom Kapital als ökonomisch vermitteltem Herrschaftsverhältnis und als ,automatischem Subjekt" der kapitalistischen Ökonomie. Weiter wurde gezeigt, wie diese Bewegung ihre

2 a) Vgl. E. Altvater, J. Hoffmann, W. Schoeller, W. Semmler, Entwicklungstendenzen im Kapitalismus Westdeutschlands (I und II) in: PROKLA Nr. 13 und 16 (1974); E. Altvater, J. Hoffmann, R. Künzel, W. Semmler, Inflation und Krise der Kapitalverwertung, in: PROKLA 17/18 (1975) 
eigenen Schranken produziert und Widersprïche in der Utberakkumulation von Kapital und im tendenziellen Fall der Profitrate in den 60er Jahren manifest werden. Zugleich wurden die historischen Inhalte der Kapitalbewegung, die Niederlage der Arbeiterklasse im Faschismus und 2. Weltkrieg als Voraussetzung der Prosperitäts-Phase in Westdeutschland und die Entwicklung und Veränderung der Klassenverhältnisse mit der Entwicklung des Kapitals deutlich gemacht. (3)

Es wurden Tendenzen in der Akkumulation des Kapitals in den 50er und 60er Jahren beschrieben, die auch für die Bedingungen der Akkumulation in den 70er Jahren in Westdeutschland von Bedeutung sind: Danach schwanden die außergewöhnlich günstigen Produktionsbedingungen des Kapitals nach dem Zweiten Weltkrieg (niedrige organische Zusammensetzung, hohe Mehrwertrate aufgrund niedriger Löhne / in dem Maße, wie das Kapital die industrielle Reservearmee absorbierte und dami der äußere Druck auf die Lohnentwicklung schwand. Die Arbeiter konnten aufgrund der Knappheit der Ware Arbeitskraft höhere Löhne und kürzere Arbeitszeiten durchsetzen. Eine Steigerung der Mehrwertrate war unter diesen Umständen nur noch möglich, indem die Produktivkraft der beschäftigten Arbeiter erhöht wurde: dies war aber mit großen Kapitalinvestitionen verbunden, die zumindest die technische Zusammensetzung, aber langfristig auch - aufgrund mangelnder Entwertung, Erhöhung des staatlichen Anteils an der Produktion usw. (4) - die organische Zusammensetzung des Kapitals steigen ließ. Dieser Prozeß mußte dann zu einem Fall des Verwertungsgrads des Kapitals führen, als die Steigerung der Arbeitsproduktivitatt (also letztlich der Mehrwertrate) nicht mehr die angestiegene organische Zusammensetzung in ihren Wirkungen auf die Profitrate kompensieren konnte und zudem noch Lohnsteigerungen dem Anstieg der Mehrwertrate entgegenwirkten: die Profitrate sinkt - unterbrochen durch kurze Aufschwungphasen nach den Krisen - seit Ende der fünfziger Jahre, während zugleich der progressive Einsatz von Kapital Bedingung der Steigerung der Produktivität der Arbeit und der steigenden technischen Zusammensetzung des Kapitals seit dieser Zeit ist.

Ein wichtiger Indikator war bei diesen Analysen die Entwicklung der in der Statistik dokumentierten Kapitalproduktivität, die das Verhältnis von lebendiger zu vergangener Arbeit indizierte. Ein Fall der Kapitalproduktivität deutete auf einen Anstieg der technischen Zusammensetzung des Kapitals hin. Diese Entwicklung der Kapitalproduktivität konnte aber weiter aufgeschlüsselt werden als Verhältnis von Arbeitsproduktivität und Kapitalin tensität. Steigt die Kapitalintensität schneller als die Arbeitsproduktivität, muß die Kapitalproduktivität fallen. Andererseits eignet sich der Kapitalist resp. das Kapital nicht das gesamte Wertprodukt $(v+m)$ an. Der

3) Dies ist allerdings aufgrund notwendiger Beschränkungen in den o.a. Artikeln nur angedeutet worden. Wir werden unten versuchen, gerade die Erfahrung der Vollbeschäftigung im Zusammenhang mit der Kritik an der gewerkschaftlichen Politik gegenüber der Arbeitslosenproblematik zu diskutieren.

4) Vgl. zu einem Versuch der theoretischen Erklärung des Falls der Profitrate aus diesem Zusammenhang Altvater u.a., Inflation und Krise der Kapitalverwertung, a.a.O., S. $255-262$ 
Zähler des Ausdrucks für die Kapitalproduktivität mußte somit noch um den Anteil, der zur Reproduktion des Arbeiters dient (dem Lohnanteil), verringert werden. Erst durch diese Berechnung wurde ein der Profitrate entsprechender Aus druck entwickelt, dessen empirisch nachvollziehbare Tendenz den Verlauf der Profitratenentwicklung anzeigt. (5)

Im folgenden wollen wir nun die Entwicklung dieser Indikatoren für die Profitrate für die 70er Jahre verfolgen, um aus der Bewegung dieser Indikatoren Folgerungen für den Verlauf der Akkumulation und für die Ursachen der Arbeitslosigkeit ziehen zu können. Anschließend werden wir anhand der Darstellung des Žyklus 1966-1974 (5 a) den Zusammenhang von allgemeiner und periodischer Überakkumulation von Kapital entwickeln, um so die konkreten Gründe des Hervortretens einer industriellen Reservearmee herauszufinden.

Fassen wir zunächst einmal die in PROKLA 16 als Bedingungen der Kapitalakkumulation Anfang der 70er Jahre beschriebenen Tendenzen zusammen: (6)

(1) Aufgrund des internationalen Konkurrenzzusammenhangs, des herrschenden Mechanisierungsgrades und des herrschenden Lohnniveaus wird sich (bei ausgeschöpften Arbeitsvolumen) eine weitere Kapitalintensivienung nicht o. w. vermeiden lassen: Arbeitssparende Techniken werden bei der Anlage von Kapital vorherrschen, die organische Zusammensetzung wird weiter - wenn auch, wie noch darzustellen sein wird, leicht abgeschwächt - steigen.

(2) Die Arbeitsproduktivität wird aufgrund der Ausschöpfung der Produktionspotenzen, tendenziell sinkender Kapazitätsauslastung und Widerstand der Arbeiter gegen eine weitere Intensifikation der Arbeit nicht in einem solchen Maße steigen, daß sie die Wirkungen der Kapitalintensivierung auf die Kapitalproduktivität kompensieren könnte.

(3) Die Lohnentwicklung wird aufgrund der verstärkten Auseinandersetzungen an der Basis der Gewerkschaft und in den Betrieben, wie sie sich Ende der 60er Jahre ankündigten, den Druck auf die Profitrate nicht entlasten, eher dürfte von dieser Seite eine Einschränung des akkumulierbaren Profits erfolgen.

Zwar können wir hier nicht die Untersuchung in PROKLA 16 fortführen, da daß dazu notwendige statistische Material, das sich mit dem dort verwandten vergleichen ließe, noch nicht vorliegt; andererseits gibt es Hinweise auf die Entwicklung

Vgl. folgende Formel für die Kapitalrentabilität $\pi$

$\pi=\frac{\text { Arbeitsproduktivität }}{\text { Kapitalintensität }}(1-$ Lohnquote $)=\frac{\frac{Y}{L}}{\frac{K}{L}}(1-w)$

(wobei der Bruch auch als ,Kapitalproduktivität“" gilt!)

5 a) Wir meinen, daß sich die Annahme eines 4-5jährigen Zyklus nicht stützen läßst. Vielmehr ist ein 7-8jähriger Zyklus mit einer ,Zwischenkrise" für die BRD-Entwicklung eher typisch. Darauf kann hier aber nicht näher eingegangen werden.

Vgl. Altvater u.a., Entwicklungstendenzen . . a.a.O., S. 137-149 
der Kapitalproduktivität seit 1971. So spricht der „Sachverständigenrat zur Begutachtung der gesamtwirtschaftlichen Entwicklung" in seinem letzten Jahresgutachten (1974) von einer sìnkenden Kapitalproduktivität: „Gegenwärtig dürfte die potentielle Kapitalproduktivität jährlich um $1 \mathrm{vH}$ bis $11 / 2 \mathrm{vH}$ sinken. " (7) Daraus wird gefolgert: „Soll sich das Wachstum nicht noch weiter verlangsamen, so müssen, bei gleichbleibender Abnahme der Kapitalproduktivität, die Nettozugänge zum Kapitalstock mittelfristig um $41 / 2 \mathrm{vH}$, die Bruttoinvestitionen sogar um $51 / 2 \mathrm{vH}$ zunehmen." Sieht man einmal davon ab, daß eine solch lineare Tendenz natürlich nicht einfach zu postulieren ist - schließlich schafft ja - wie auch 1966/67 geschehen - die Krise veränderte Bedingungen der Akkumulation -, so heißt dies doch zunächst nichts anderes, als daß aufgrund der gewachsenen und weiter wachsenden organischen Zusammensetzung des Kapitals ein stets wachsender Kapitaleinsatz notwendig ist, um eine gleichbleibende Zunahme des Nettoprodukts zu bewirken.

Da das Nettoprodukt in der amtlichen Statistik in konstanten Preisen gemessen wird, kann sich das in ihm ausgedrückte Wertprodukt $(v+m)$ bei gleichbleibendem Arbeitsvolumen in Preisen eines bestimmten Basisjahres nur dann erhöhen, wenn durch eine Steigerung der Produktivität der Arbeit die produzierte Produktmenge erhöht wird. Dies erfordert aber unter den Bedingungen, die sich in Westdeutschland entwickelt haben, den forcierten Einsatz von Kapital. Die Erhöhung der organischen Zusammensetzung ist dann Ausdruck der Steigerung der Produktivkraft der Arbeit durch die Anlage neuer Maschinerie, Das Fallen der Kapitalproduktivität drückt somit aus, daß gesamtwirtschaftlich die Erhöhung der Arbeitsproduktivität nicht die negativen Effekte der Steigerung des Kapitaleinsatzes im Verhältnis zur lebendigen Arbeit auf die Profitrate hat ausgleichen können.

Können also aus diesem Zusammenhang schon Schlüsse auf eine Fortsetzung des Trends des fallenden Verwertungsgrads auch in der ersten Hälfte der 70er Jahre gezogen werden, so wird eine solche Annahme noch erhärtet, wenn die Lohmentwicklung hinzugezogen wird. Mit Ausnahme des Jahres 1972 konnten nämlich die Arbeiter ihre "Reallohnposition" von 1970 bis 1973 verbessern: Aufgrund des uberaus starken Booms Ende der 60er Jahre war die Ware Arbeitskraft Anfang der 70er Jahre äußerst knapp geworden; hinzu kam, daß so nicht nur die objektive Stellung der Arbeiter gestärkt war, sondern - ausgehend mit den Septemberstreiks 1969 - die Kampfkraft der Arbeiter gestiegen war und der Druck der Mitgliederschaft auf die gewerkschaftliche Lohnpolitik sich verstärkte.

Zudem waren die Arbeiter inflationsbewußter geworden: die stille Umver-

7) Vgl. Sachverständigenrat zur Begutachtung der gesamtwirtschaftlichen Entwicklung, Jahresgutachten 1974, Ziff. 222; Im Gegensatz zu der DIW-Statistik (Krengel u.a., Produktionsvolumen und Produktionspotential... Berlin 1972 und 1973) wird beim Sachverständigenrat in Y der potentielle Output, nicht der - durch Auslastungsschwankungen geprägte - tatsächliche Output ausgedrückt! Angesichts der sinkenden Auslastungen in den 70er Jahren dürfte die Kapitalproduktivität also tatsächlich noch stärker gesunken sein. 
teilung über die Inflation - auch von Seiten des Kapitals ein prekäres und keineswegs ,bewußt einzusetzendes Mittel" - gelang nicht mehr, obwohl die Inflationsrate seit 1969 schnell anstieg. So verbesserte sich vorerst Anfang der 70er Jahre die Reallohnposition. (8) Die Profitrate mußte daher auch von der Entwicklung der Löhne her unter Druck geraten sein, wenngleich sich darin lediglich eine Korrektur der zuungunsten der Arbeiter in Aufschwung veränderten Verteilungsverhältnisse ausdrückte.

Eine solche Entwicklung zeigt sich auch in Berechnungen des Sachverständigenrats, wenn er eine fallende Realrendite konstatiert (vgl. Schaubild 1 (9)). Auch die sinkenden Investitionsquoten drücken diesen Trend in der Verwertung aus (wenngleich im Schaubild 1 stark überzogen, insofern dort die Investitionen auf den Umsatz bezogen wurden).
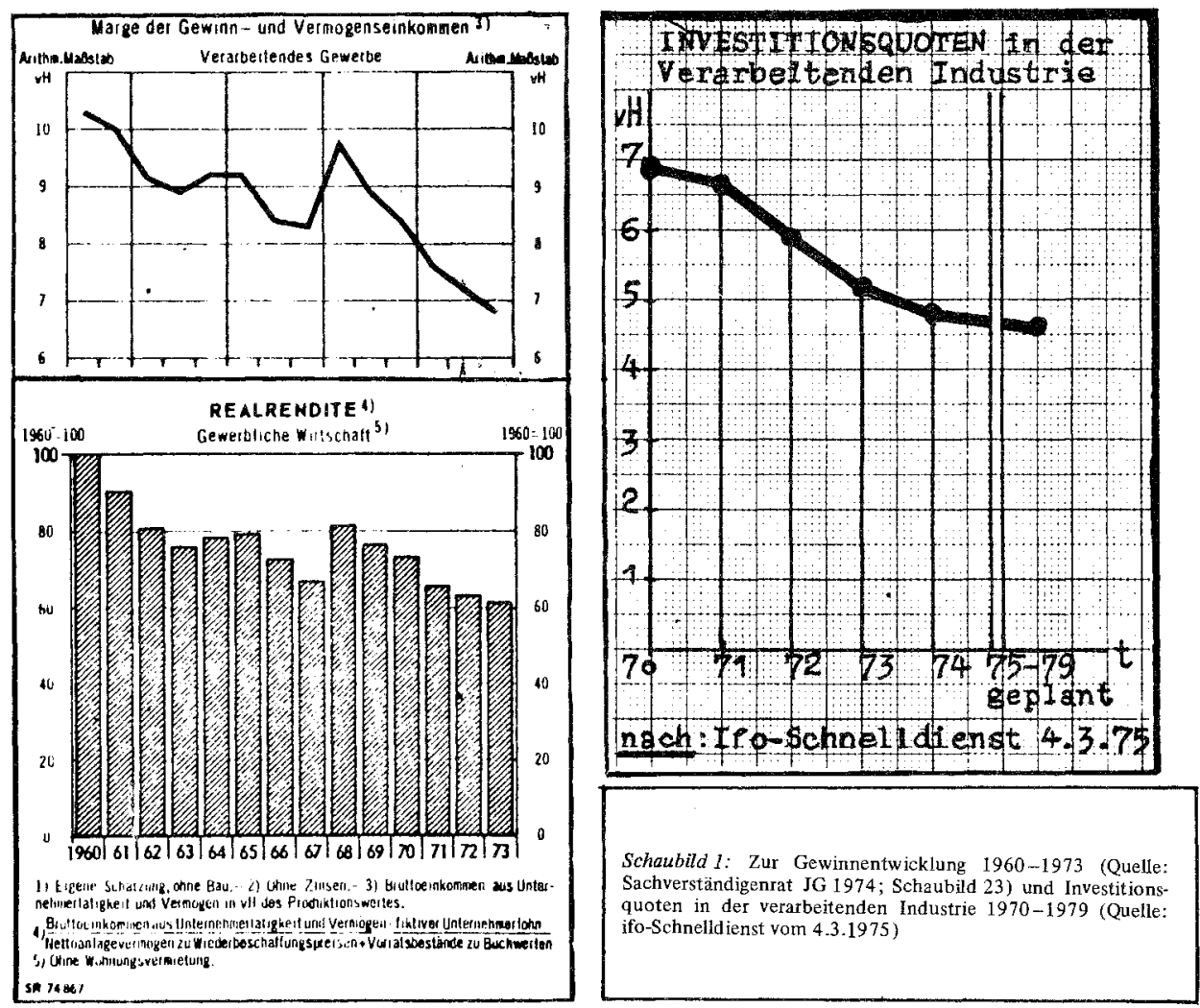

8) Dabei ist u.a. zu berücksichtigen, daß der SVR, indem er ein kostenniveauneutrales Wachstum der Löhne dem tatsächlichen Wachstum gegenüberstellt, das Bruttoinlandsprodukt je Enwerbstätigen zum Ausgangspunkt nimmt, um den Lohnspielraum der Arbeitnehmer zu berechnen. Vgl. JG 1974, Tab. 12 (im Text).

9) Der Fall der Realrendite kann aber nur als ein sehr oberflächliches Indiz hier gelten! Wichtiger sind in diesem Zusammenhang die anderen angeführten Indikatoren. 
Die von uns anhand von Indikatoren der amtlichen Statistik konstatierte steigende organische Zusammensetzung des Kapitals muß sich aber auch in der langfristigen Entwicklung der Abteilungen der industriellen Produktion zueinander darstellen (und dies wird - wie wir später sehen werden - von großer Bedeutung für die langfristige Entwicklung der Arbeitslosigkeit in Westdeutschland sein.)

Denn die von uns behauptete steigende organische Zusammensetzung des gesellschaftlichen Gesamtkapitals müß sich auch in einem unterschiedlichen Wachstum der beiden Abteilungen der gesellschaftlichen Produktion, dem Produktionsmittelsektor und dem Konsumgütersektor, ausdrücken. Ein steigender Anteil konstanten Kapitalteils gegenüber dem variablen Teil bedeutet schließlich eine schnellere Zunahme der Produktionsmittelproduktion gegenüber einem geringeren Wachstum der Produktion von Konsumgütern. Ein schnelleres Wachstum der Produktionsmittelabteilung wollen wir anhand von zwei Indikatoren für die Bundesrepublik nachweisen: des Wachstums des Beschäftigungsanteils in der Investítionsgüterindustrie gegenüber dem in den Verbrauchsgüterindustrien und der Strukturveränderung innerhalb des Nettoproduktionsvolumens.

a) Aus Schaubild 1a geht deutlich hervor, daß der Anteil der geleisteten Beschäftigungsstunden der Investitionsgüterindustrien gegenüber den Anteilen der anderen Industriebereiche stark angestiegen ist. Dies gibt eine Verschiebung der Verteilung der gesellschaftlichen Arbeit zugunsten der Abteilung I an, soweit diese Abteilung durch die Investitionsgitterindustrien (Maschinenbau, Fahrzeugbau, Elektroindustrie) repräsentiert wird. Auch wenn zu den Investitionsgüterindustrien die Grundstoff- und Produktionsgüterindustrie hinzugezählt werden (da auch sie zur Abteilung I gehören), verändert sich - da dort die Beschäftigung stagniert - das Bild nicht! Die Stagnation der Beschäftigung im Bereich Grundstoff- und Produktionsgüterindustrien ist dabei wesentlich auf die überproportionale Produktivitätssteigerung in diesem Sektor zurückzufuhren. 
$\mathrm{vH}$
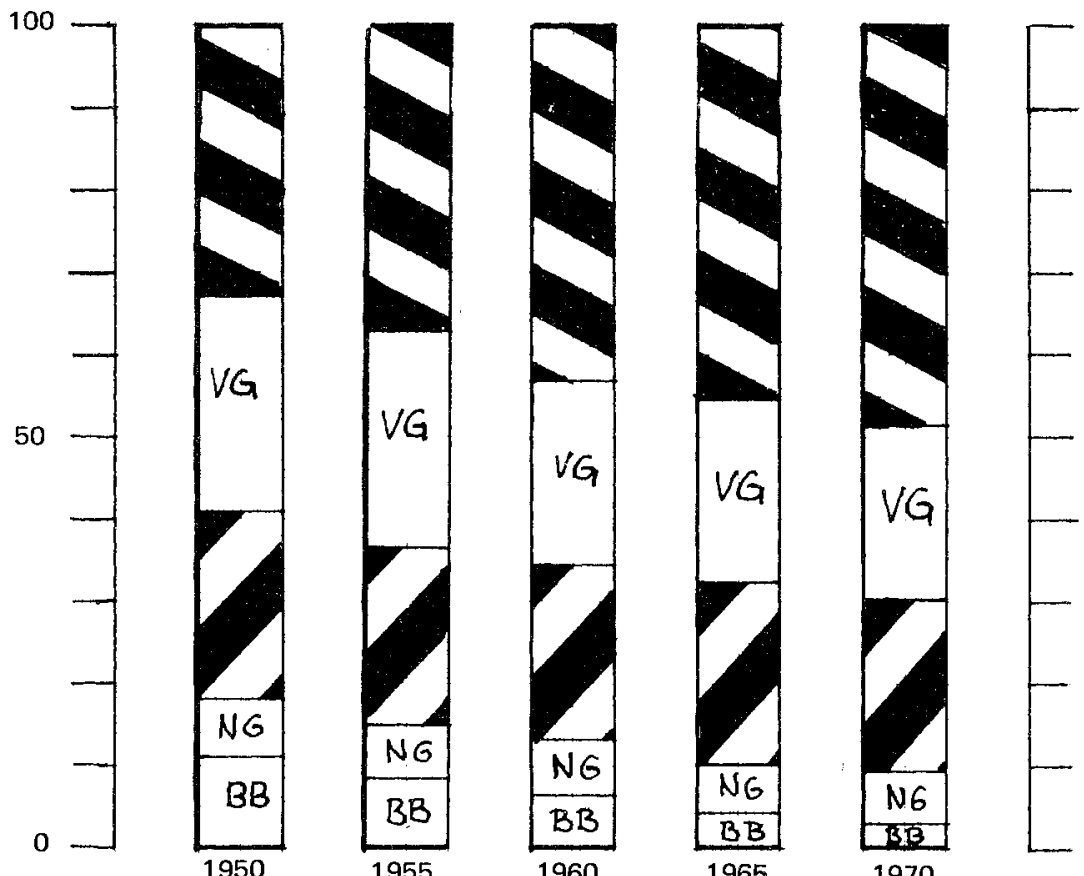

1965

1970

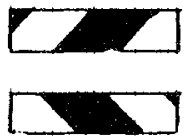

Grundstoff- $u$. Prod.güter Investitionsgüterindustrie.

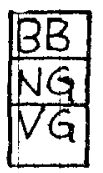

Bergbau

Nahrungs-/G.

Verbrauchs-

güterindustrie

Schaubild 1a: Anteile ausgewählter Industriegruppen an den in der Industrie insgesamt geleisteten Beschäftigungsstunden (in $\mathbf{v H}$ )

Quelle: $\quad$ R. Krengel u.a., Produktionsvolumen und Produktionspotential ... a.a.O., 13., 14., 15. Folge, Berlin (W) 1973 ff., S. 22 
b) Dieselbe Tendenz läßt sich nun an der Veränderung der Struktur des Nettoproduktionsvolumens ablesen. Hier steigt nach Untersuchungen des DIW (9a) der Anteil des Nettoproduktionsvolumens der Investitionsgüterindustrie von 1950 bis 1970 von $27,5 \mathrm{vH}$ auf $36,2 \mathrm{vH}$; demgegenüber vermindert sich der Anteil der Verbrauchsgüterindustrie im selben Zeitraum von $20,5 \mathrm{vH}$ auf $18,3 \mathrm{vH}$. Der Anteil der Grundstoff- und Produktionsgüterindustrien steigt leicht an. Da die Produktivitätszunahme in der Investitionsgüterindustrie durchschnittlich war, korrespondiert hier unmittelbar die Produktionszunahme mit der Zunahme der Arbeitskräfte, was die überproportionale Steigerung der Abteilung I als gesichert erscheinen läßt und unsere These einer gestiegenen organischen Zusammensetzung in bezug auf die Abteilungen der gesellschaftlichen Produktion für die Bundesrepublik stützt!

Allerdings könnte diese Au'sweitung gleichermaßen einer Ausweitung des Exportanteils der Investitionsgüterindustrieproduktion zuzuschreiben sein. Dieser ist mit 1960: $53 \mathrm{vH}$ und 1970: $58 \mathrm{vH}$ traditionell in der Bundesrepublik hoch, wiegt jedoch nicht - wie aus den Zahlen deutlich wird - die überproportionale Steigerung des Anteils der Investitionsgüterproduktion im Inland auf. (9 b) Zugleich zeigt sich anhand dieser Daten die hohe Abhängigkeit der Investitionsgüterindustrie vom Welt-, markt (10).

Aus unserer bisherigen Darstellung, so können wir zusammenfassen, ergeben sich für die weitere Analyse des Akkumulationsprozesses in der Bundesrepublik drei Strukturnerkmale der langfristigen Entwicklung, die sich auch für die Herausbildung der Arbeitslosigkeit relevant erweisen werden:

(1) Die Akkumulation des Kapitals führte in der Bundesrepublik zu einer steigenden organischen Zusammensetzung und einem Fall der Profitabilität.

(2) Diese beschleunigte Akkumulation führte zu einem überproportionalen Wachstum der Abteilung I (hier besonders der Investitionsgüterindustrien).

(3) Die traditionell hohe Exportabhängigkeit gerade dieser Industrien wurde in den 60 er Jahren noch größer.

Insgesamt wird so deutlich, daß der Überakkumulation von Kapital auch eine Überexpansion der Abteilung I entspricht. Andererseits muß sich Überakkumulation und Überexpansion der Abteilung I (was denselben Sachverhalt ausdrückt) auch in der Krise als Überproduktion von Waren darstellen. Um diesen Zusammenhang klärẹn zu können, müssen wir hier allerdings die Ebene der "Trendanalyse“ verlassen und untersuchen, in welcher Weise sich die Überakkumulation von Kapital im Zyklus darstellt. Dadurch können wir Trendentwicklung und zyklische Bewegung miteinander vermitteln und die Ursachen der Arbeitslosigkeit aufzeigen.

9 a) R. Krengel u.a., Produktionsvolumen und Produktionspotential, Stat. Kennziffern, 13. und 15. Folge, Berlin 1973 und Berlin 1974

9 b) Vg1. Ifo-Institut, Strukturdaten über die Industrie, 3. Folge 1960-1972, München 1974, Tabelle 4

10) Die Investitionsquote ist vom Ifo-Institut als „Anteil der Bruttoinvestitionen am Gesamtumsatz" berechnet worden und daher stark umsatzabhängig. Th. Brinkmann vom Institut der Deutschen Wirtschaft (!) rechnet übrigens eine - gemessen an den durchschnittlichen Zuwachsraten der Investitionen 1960-1970 ,fiktive“ Investitions- 


\section{Erover \\ $48+30-3$}

Die hier kurz entwickelten Tendenzen der Akkumulation und der sektoralen Entwicklung sind also zunächst nur als Trend zu verstehen, der sich in einem längeren historischen Zeitraum durchsetzt und - wie wir sehen - auch für die 70er Jahre, gilt. Entgegenwirkende Tendenzen sind in diesem Trend nicht mehr sichitbar, sondern im Resultat aufgehoben. Insbesondere ist hierin ja gerade von den aus der zyklischen Kapitalakkumulation hervorgehenden ,bereinigenden“ Einflüssen auf die Profitrate abstrahiert, wie andererseits auch die Profitrate als Triebkraft des Zyklus nicht mehr sichtbar wird. Daher müssen wir hier den Zusammenhang von langfristiger und zyklischer Akkumulation anhand des historischen Materials noch näher herausarbeiten.

Bei einer solchen Untersuchung ist es wichtig, nicht nur das Kapital als vorgeschossenes Geldkapital aufzufassen, das sich zu einer bestimmten Profitrate verwerten muß; hinsichtlich der zyklischen Überakkumulation ist es vielmehr bedeutsam, das Kapital auch als produktives Kapital als akkumulierte „Produktionspotenz"; als Kapital in stofflicher Form zu betrachten. Denn mit der Kapitalakkumulation wächst nicht nur das vorgeschossene Geldkapital an, sondern es findet auch eine Anhäufung von produktivem (fixen) Kapital statt, dessen Anwendung und Auslastung von der kontinuierlichen Realisierung des produzierten Warenkapitals, kurz: von der Nachfrage abhängt. (11) Die Überakkumulationskrise muß daher nicht nur als Uberakkumulation von Kapital in seiner Wertform, sondern auch als periodische Überakkumulation von produktivem Kapital in seiner stofflichen Form entwickelt werden. Die verschiedenen Kapitalformen dürfen also bei der Betrachtung des langfristigen Akkumulationsprozesses und seiner zyklischen Verlaufsform nicht woneinander getrennt werden - wie dies in gängigen Krisenerklärungen der $\mathrm{Fall}$ ist.

Wie ist nun dieser Prozeß zu beschreiben, der im Verłauf des Akkumulationsprozesses sowohl eine absinkende Profitrate als auch eine Überakkumulation von produktivem Kapital und eine Überproduktion yon Waren hervorbringt?

a) Betrachten wir zunächst die Komponenten für die Bewegung der Profitrate seit der letzten Krise 1966/67 in Westdeutschland. Die Krise als bestimmte Phase im

lücke 1971-1974 von 100 Mrd. DM aus, was ebenfalls den Rückgang der Investitionen veranschaulicht. Vgl. T. Brinkmann, Wachstums- und Verteilungsspielraum 1975-1980, Köln 1975, S. 26 f.

11) Vgl. dazu bei Marx die Ausführungen über die Wirkungen des produktiven Kapitals, z. B. Karl Marx, Das Kapital, Bd. II, Berlin (DDR) 1963, S. 357 und ders., Das Kapital, Bd. III, Berlin (DDR) 1964, 15. Kapitel. Der folgende empirisch darzustellende Zusammenhang von Überakkumulation und Überproduktion wurde theoretisch genauer von uns in „Kapitalakkumulation, Staatseingriffe und Lohnbewegung“" in: PROKLA Nr. 2 (1972), S. 20-26 entwickelt. 


\section{$66 / 67$ Len}

industriellen Zyklus stellte sich zunächst als eine tiefgehende ${ }_{2}$ Bereinigung " der Verwertungsschwierigkeiten des Kapitals dar. Als Wirkung der Krise, durch den Abbau unproduktiver Kapazitäten, die Kapitalvernichtung und -entwertung durch Konkurse, relativen Preisfall und Konzentration/Zentralisation, den Druck auf den Lohn, verbesserten sich die Verwertungsbedingungen des Kapitals erneut. Anhand des Gutachtens des Sachverständigenrats wurde an anderer Stelle bereits im einzelnen dieser Prozeß beschrieben. (12) Mit der langsam sich ausdehnenden und durch staatliche Konjunkturprogramme und Weltmarkt beschleunigten Nachfrage nach Produkten der Abteilung I und II im Aufschwung stieg die Auslastung des yorhandenen produktiven Kapitals. Hierdurch wie auch durch den Einsatz zusätzlichen fixen Kapitals im Produktionsprozeß wurde die Arbeitsproduktivität angehoben. Zugleich fanden neue technologische Verfahren in der Produktion Anwendung, die den Konkurrenzkampf im Inland und auf dem Weltmarkt erleichterten. (13) So läßt sich im Aufschwung $1968 / 69$ feststellen, daß die (in jeweiligen Preisen ausgedrückte) Mehrwertrate (14) als Folge zunehmender Auslastung des produktiven Kapitals, zunehmender Kapitalintensivierung und Arbeitsproduktivität bei nachhinkenden Löhnen ansteigt. Auch die Profitrate hebt sich wieder - trotz angewachsener organischer Zusammensetzung des Kapitals - über das in der Krise festgesetzte Niveau. (15) Im weiteren Verlauf des Aufschwungs und dos Booms wurden die (auch vom Sachverständigenrat 1969 hervorgehobenen) stillen Reserven der Steigerung der Arbeitsproduktivität aber ausgeschöpft: So sinken die Zuwachsraten der (gesamtwirtschaftlichen) Ar beitsproduktivität von $+7,4 \mathrm{vH}(1968)$ auf $+2,9 \mathrm{vH}(1971)$. (16) Zugleich wurde wieder der Arbeitsmarkt leergefegt und die Stellung der Arbeiter gegenüber dem Kapital stärker. Das Zurückbleiben der Arbeitsproduktivität und das Ansteigen der Löhne erzwangen den Ubergang zu kapitalintensiveren" Methoden der Produktion, wodurch vermutlich auch die organische Zusammensetzung des Kapitals weiter anstieg (vgl. Pkt. b). Die Durchsetzung hoher Geldlohnforderungen der Lohnarbeiter 1970 und 1971 kompensierten in dieser Phase das weite Zurückfallen der Lohnzunahmen hinter den Zuwachsraten der Profite im Aufschwung, andererseits beschränkten sie zu einem Zeitpunkt die Profitmasse des Kapitals, in dem die Profitrate bereits aufgrund der oben geschilderten Entwicklung unter Druck geriet. In derselben Phase des Zyklus stiegen zusätzlich - als

12) Entwicklungstendenzen ... (II), a.a.O., S. 127 f. Konzentration, Zentralisation, die Einführung neuer Produktionsmethoden, neuer Arbeitsorganisation auf der Basis der Entwertung und Vernichtung von Kapital in der Krise werden vom Sachverständigenrat dort als ,reinigende Funktion der Krise" verstanden.

13) Sachverständigenrat zur Begutachtung der gesamtwirtschaftlichen Entwicklung, Jahresgutachten 1969, Ziff. 50-54

14) Zum Problem des Verhältnisses von Lohnquote und Mehrwertrate vgl. Entwicklungstendenzen II, a.a.O., S. 114 ff.

15) Im Schaubild 3 in Entwicklungstendenzen II, a.a.O., wird der zyklische Verlauf der Profitrate (soweit diese von der Kapitalrentabilität indiziert wird) sichtbar.

16) Sachverständigenrat, Jahresgutachten 1974, Tabelle 12 (im Text) 
Folge einer verschärften Konkurrenz der industriellen Kapitale um Kredit und als Folge einer restriktiven Zentralbankpolitik - die Zinssätze und beschränkten von dieser Seite her die Profitmasse des industriellen Kapitals. (17) Zugleich stiegen die Kosten fur das zirkulierende konstante Kapital : die Rohstoffe verteperten sich ab 1971/72. Diese Veränderungen in den Verwertungsbedingungen hatten ifsgesamt ein Abfalten der Profitabilitäf des Kapitals zur Folge. Der Er: höhung der Profitrate im Aufschwung steht so wieder eine Tendenz zum Sinken iri Boom tentgegen

b) Zügleich stelite sich aber der Aufschwung und die beschleunigte Akkumulation noch als rapide (Anhäufung von produktivem Kapital dar, als Aufbau großer Produktionskapazitâten. Wir können diesen Ausbau der Kapazitäten im Aufschwung Ende der 60er Jahre anhand der hohen Nettozugänge zum Kapitalstock, wie sie aus dem Schaubild 2 ersichtlich werden, verfolgen.

Schaubild 2: Nettozugänge (Mrd. DM) zum Kapitalstock $1962-1975$, log. Maßstab; Quelle: Sachverständigenrat, Jahresgutachten 1974 Schaubild 31

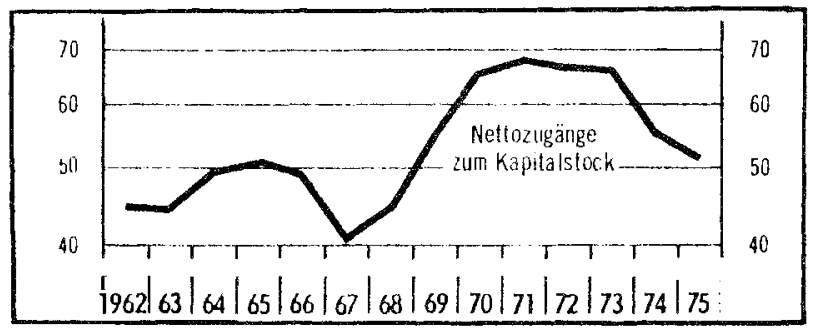

Die hohen Zuwachsraten des Kapitalstocks sind zum einen Ergebnis des explosiven Investitionsbooms der Jahre des Aufschwungs, zum anderen drickt sich darin die Notwendigkeit aus, daß ein steigender Zusatzkapitaleinsatz zur Produktion des Profits und der Erhaltung der Wachstumsrate des. Sozialprodukts" enforderlich ist. (18) Mit dem Boom verschärften sich so nicht nur die strukturellen Verwertungsprobleme (vgl. Pkt. (a)), sondern es zeichnete sich auch eine $\ddot{U} b e r a k k u$ mulation von produktivem Kapital ab (die auch als ,disproportionale" Entwicklung in Erscheinung trat). Die ansteigende Nachfrage nach Produkten der Abt I - infolge der raschen Akkumulation und Anlage von fixem Kapital - war nicht nur durch die schnellere Ausdehnung der Abt. I gekennzeichnet, sondern es wurden auch in bejden Abtelungen der gesellschaftlichen Produktion Kapazitäten in Form von produktivem Kapital aufgebaut, deren Anwendung und Auslastung auch von der kontinuierlichen Ausdehnung der Nachfrage abhängt. Dabei verstärkte die Anwendung neuer technischer Verfahren in den beiden Abteilungen die Produktionspotenzen ebenso wie die Rationalisierung und die Intensivierung der Arbeit.

17) Vgl. Sachverständigenrat, JG 74, Schaubild 29

18) Vgl. auch Sachverständigenrat, JG 1974, Ziff. 22 
Daraus ergeben sich aber Kansequenzen für die Bewegung der Profitrate im Zyklus: Hat die Neuanlage von fixem Kapital im Aufschwung und im Boom eine Erhöhung der technischen und organischen Zusammensetzung des Kapitals zur Folge, mit der Konsequenz einer überproportionalen Entwicklung der Abteilung I, so bleibt dies doch noch ohne bedeutsamen Einfluß auf die Bewegung der Profitrate, solange die Profitmassen durch die Auslastung des produktiven Kapitals und die Produktivitätssteigerungen infolge günstiger Nachfrageverhältnisse noch gehalten oder sogar gesteigert werden können. Nur wenn also auch eine hinreichende Nachfrage yorhanden ist, wenn die Realisierungsbedingungen noch gut sind, können die günstigen Verwertungsbedingungen in der Produktion noch ausgenutzt und die aktuellen Profitraten von den Einzzlkapitalen noch gehalten werden. Auf diese Weise hängt die Profitrate also auch von der Nachfrage, den Realisierungsbedingungen (nicht zuletzt auf dem Weltmarkt) und der davon abhängigen Auslastung des produktiven Kapitals ab! Aber: Umgekehrt hängt die Nachfrage wiederum von der erwarteten Profitrate ab. Dies zeigt sich auch in der folgenden Phase des Zyklus:

c) Denn gerade die beschleunigte Neuanlage von produktivem Kapital stäßst ab 1970 andrenzen

- Die vorangegangene Kapitalakkumulation hat te die erwartete Profitabilität neuer Zusatzkapitale stark herabgedrückt, da die gestiegene organische Zusammensetzung nicht mehr durch hohe Zuwachsraten der Arbeitsproduktivität (und letztlich der Mehrwertrate) kompensiert wurde und andererseits die (nachzuholenden) Lohnsteigerungen 1970/71 zusätzlich die Mehrwertmasse und damit die Profiterwartungen einschränkten. Seit 1969 sinken die Wachstumsraten der Arbeitsproduktivität, 1970 können die Gewerkschaften die höchsten Lohnsteigerungen in der Geschichte der BRD durchsetzen; zugleich steigen die Nettozugänge zum Kapitalstock (mit der Folge eines verschärften Konkurrenzkampfes) noch bis 1971 an.

- Zugleiçh zeichnet sich Anfang der 70er Jahre eine Überakkumulation von Kapital in produktiver Form ab: Die Anhaufung von Produktionskapazitäten, fixem Kapital, stößt auf beschränkte Absatzmöglichkeiten. Dies ist in der Phase des beginnenden Abschwungs dadurch der Fall gewesen, daß die Wachstumsraten der Nachfrage nach Produkten .der Abt. I (Güter der Grundstoff-, Produktionsmittel- und Investitionsgüterindustrie) aufgrund sinkender Profiterwartungen in beiden Abteilungen der Produktion zurückging. Andererseits wurde in der Abt. II (Konsumtionsmittel) durch die Anlage von produktivem Kapital der ,potentielle Output" derart erhöht, daß zumindest keine großen (wie wir wissen „profitmindernden") Zusatzanlagen mehr notwendig waren, als 1972/73 in einem ,Zwischenhoch" des Zyklus als Folge der Lohnerhöhungen die Verbrauchsnachfrage wieder anzog. Neben dieser Stützung der Nachfrage durch den privaten Verbrauch war es nun allerdings auch die starke Nachfrage des Auslandes und das Aufrechterhalten der staatlichen Nachfrage, die die Auslastung der Kapazitäten noch gewährleisteten. Kurzfristig wurde so ein Aufschwung der Produktion besonders in der Abt. II 
(Konsumgüter) ermöglicht. (19) Die aufgrund der allgemein sinkenden Gewinnerwartungen vorherrschende Zurückhaltung in der Neuanlage von produktivem Kapital (vgl. Pkt. a) führte zunächst zu einer Stagnation in der Abt. I (20). Deren Auslastung wurde im wesentlichen durch die Nachfrage des Auslands nach westdeutschen Investitionsgütern getragen, (21) während die Inlandsnachfrage stagnierte und 1973/74 schnell zurückging. Statt ihr produktives Kapital auszuweiten, nutzten die Kapitalisten (in beiden Abteilungen) in der kurzen Phase der wieder angestiegenen Nachfrage weitgehend nur ihr $>$ vorhandenes produktives Kapital voll aus (vgl. Schaubild 3). (22) Die Grenze, die sich hier für die Neuanlage von produktivem Kapital zunächst stellte, war also die sinkende Profiterwartung in beiden Abteilungen aufgrund des vorhandenen Produktionspotentials.

19) So steigen die Staatsausgaben wie folgt (jährl. Veränd.): 1968: + 2,3;1969: +9,8;1970: $+12,5 ; 1971$ : $: 15,7 ; 1972:+11,7 ; 1973:+12,5$ (in vH); wichtig auch die Zunahme der Konsumentenverschuldung: 1970: 27,24 Mrd. DM; 1973: 46,44 Mrd. DM! Zur Auslandsnachfrage vgl. JG 73, Ziff. 108: „Die ausländische Nachfrage nach Produkten der westdeutschen Investitionsgüterhersteller (war) 1973 außerordentlich stark..., während sich die heimischen Investoren nach dem Stabilitätsprogramm mehr Zurückhaltung auferlegten." $A$. Blechschmidt folgert daraus, daß die inländische Investitionskonjunktur zugunsten der ausländischen abgekoppelt (1) wurde: "Die Vollauslastung war schon im Früjahr 1973 erreicht, die Gewinne steigen folglich auch steil an, aber es fehlte schlicht und einfach an den entsprechenden Maschinen, die mit den Gewinnen hätten gekaut1 werden $k$ önnen. Da sie exportiert wurden, weil im Ausland - infolge der stärkeren Inflation - die besseren Geschäfte gemacht werden konnten, hätten die BRD-Kapitale die Exporte von Maschinen etc. nur dann erfolgreich in der BRD zurückhalten können, wenn sie um so höhere Preise geboten hätten. Da natürlich die investierenden Kapitale die Preise ihrer eigenen Produkte erhöht hätten, wären damit Ietztlich die Konsumgüterpreise noch mehr gestiegen, so daß wahrscheinlich die vielen Einzelstreiks um Teuerungszulagen in einen gemeinsamen Streik eingemündet wären." (Kritisches Jahrbuch 1974, Ffm 1974, S. 20). Soviel Rücksicht auf mögliche Streiks sollte man allerdings den Kapitalen nicht zutrauen, zumal die Kapitalisten im Anschaffen von Maschinen sich bestimmt nicht um mögliche Preiserhöhungen von Konsumgütern kümmern, wenn diese Zusatzanlage nur profitabel zu sein verspricht: Genau dies ist aber eben zu diesem Zeitpunkt nicht mehr der Fall! Es gibt eben keinen ,allgemein-kapitalistischen Willen", wohl aber kapitalistische Gesetzmäßigkeiten, die sich über das Handeln der Einzelkapitale durchsetzen.

20) Selbst der Sachverständigenrat führte diese Zurückhaltung auf eine absinkende Kapitalproduktivität zurück, vgl. JG 1973, Ziff. 82

21) Dabei kam dem deutschen Kapital sogar die Ölkrise äußerst zustatten, denn die „,wichtigste Rolle" im Auslandsgeschäft spielte wohl, daß die heimischen Unternehmen oft gerade jene Güter anzubieten hatten, die 1974, nicht zuletzt als Folge der Ölkrise, weltweit besonders gefragt waren. (Sachverständigenrat, JG 74, Ziff. 98) Vgl. JG 72 , Ziff. 124 ; Jg 73 , Ziff. 86 


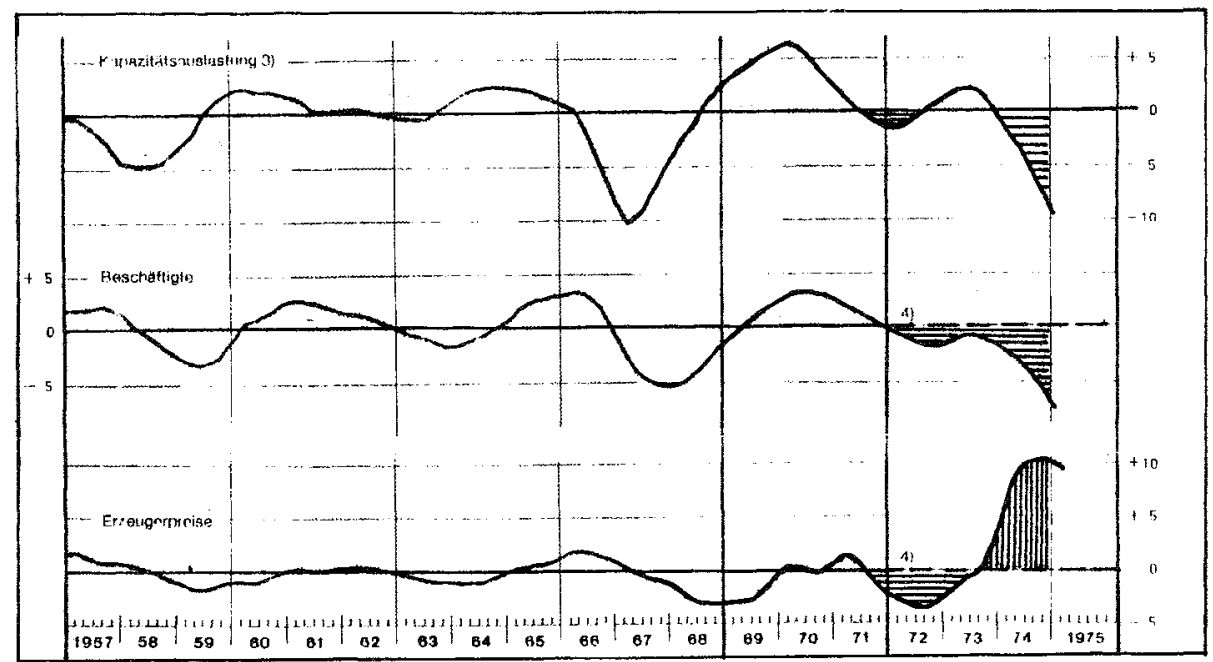

Schaubild 3: Kapazitätsauslastung, Beschäftigte und Erzeugerpreise in der Industrie der Bundesrepublik

Quelle: $\quad$ Monatsberichte der Deutschen Bundesbank Juli 1975

Ende 1973 verändern sich aber auf der Basis dieser allgemeinen Zurückhaltung in der Akkumulation die Bedingungen der Auslastung des vorhandenen produktiven Kapitals:

(1) Gegen die beschleunigte Inflation versucht der Staat (und die Bundesbank) ein restriktives Stabilitätsprogramm durchzusetzen: die staatliche Nachfғage stagniert, die Finanzierungsmöglichkeiten für Investitionen werden durch hohe Zinssätze zusätzlich eingeengt. (23)

(2) Die inflationären Preissteigerungen drücken auf die Reallöhne und in dieser Weise auf die Konsum tion der Arbeiter: die Verbrauchsnachfrage sinkt.

(3) Die Inflation schlägt auf das industrielle Kapital durch: die Kostpreise (Wiederbeschaffungspreise) steigen (vgl. Schaubild 3, Erzeugerpreise), während zugleich der Absatz sich verengt (24); die erwarteten Profite auf Zusatzanlagen sinken.

23) Allerdings sind diese hohen Zinssätze 1973/74, die den Gewerkschaften Anlaß boten, in der Bundesrepublik den Schuldigen an der Krise dingfest zu machen, nicht nur Ausdruck einer politischen Entscheidung der Bundesbank, sondern zunächst notwendige Begleiterscheinungen jeden Abschwungs. Eine Analyse des letzten Kreditzyklusses steht allerdings noch aus, vgl. aber die aufschlußreiche Untersuchung der Geldpolitik im Abschwung 1964/66 von G. Lindner, Die Krise als Steuerungsmittel, in: Leviathan Nr. 3 (1973), S. 342 ff.

24) So verteuerten sich die industriellen Produkte (also wesentlich Produkte, die wieder in die Produktion eingehen) im Frühjahr 1974 in "ungewöhnlichem Tempo" (vgl. Sachverständigenrat, JG 1974, Ziff. 119), während die Preiserhöhungsrate bei den Verbraucherpreisen nahezu stagnierte. Dies kann als ein wichtiges Indiz dafür gelten, daß zu diesem Zeitpunkt die Sicherung der „realen Akkumulation“ durch die (inflationäre) „,monetäre Akkumulation" nicht mehr gelingt. Vgl. dazu Altvater u.a., Inflation... a.a.O., S. $280-283$ 
Damit entwickelte sich ein kumulativer Prozeß nach unten: Die Krise setzte sich in wichtigen Branchen, insbesondere der Verbrauchsgüterindustrien, durch, während die Auslastung der Investitionsgutterindustrien noch durch die Weltmarktnachfrage gewährleistet war. Mit dem Rückgang der Auslandsnachfrage im Zuge der Synchronisierung des Krisenzyklus auf dem Weltmarkt $1974 / 75$ sanken die Profite auch der exportorientierten Kapitale, die bis dahin ihre Kapazitäten noch auslasten konnten. Die (entgegen den Erwartungen) verschärfte Krisensituation 1975 wird daher wesentlich von den exportorientierten „Wachstumsindustrien" (Stahl, Elektrotechnik, Chemie, Maschinenbau und Fahrzeugbau) getragen. Diese Entwicklung wird sich auch in der Struktur der Arbeitslosigkeit zeigen.

\section{d) Überakkumulation und Überproduktion von Waren im Zyklus}

Die Einschränkung der Akkumulation und die Krise 1974/75, die zunächst einhergingen mit einer Geld- und Kreditkrise, sind so Resultat der Überakkumulation von Kapital: Ubea klkamulation von Kapitalwert, der sich nicht mehr zur vorausgesetzten Profitrate verwerten kann und bbetakkamulation von produktivem Kapital, das im Verhältnis zur beschränkten Investitions- und Konsumnachfrage nur ungenügende Anwendung findet Es handelt sich hier also nicht um zwei „Fälle“ (25), sondern um die Überakkumulation von Kapitalwert und von produktivem Kapital, dessen notwendige Auslastung in Widerspruch steht zu der - bezogen auf die angehäuften Produktionspotenzen - beschränkten Nachfrage aufgrund der mangelnden Zusatzanlage von Kapital (die die Nachfrage in der Abt. I und zwischen den Abteilungen I und II betrifft) und der beschränkten Konsumtionsfähigkeit der Massen. Das Aufrechterhalten der staatlichen Nachfrage und der Nachfrage des Auslands konnte so zwar diesen Widerspruch (insbesondere für die Abt. I) noch hinausschieben, nicht aber aufheben! Ebenso war es nicht eine absolute Beschränkung der Konsumtionsfähigkeit der Massen (die Löhne stiegen sogar noch an und beschränkten daher die angeeignete Mehrwertmasse), sondern die im Verhältnis zum angehäuften produktiven Kapital begrenzte Nachfrage, die die Auslastung der Kapazitäten der Abteilung II nicht mehr gewăhrleistete und so den Fall der Profitrate auch hier verstärkte.Kern des krisenhaften Prozesses war die mangelnde Verwertung des Kapitals, die den weiteren Aufbau von Kapazitäten (und damit die weitere Nachfrage) in beiden Abteilungen verhinderte: die absinkenden Investitionsquoten (wie sie im Schaubild 1 dokumentiert sind) unabhängig von der Auslastung der Kapitale im kurzen Wiederaufschwung 1972/73 belegen dies.

Wir können nunmehr die eingangs gestellte Frage nach dem Charakter der Krise im kapitalistischen Akkumulationsprozeß beantworten. Wenn sich die Überakkumulation von Kapital als Überproduktion von Waren, unausgelasteten Kapazitäten bei den Einzelkapitalen und sinkender Zusatzanlage darstellt, so ist darin nur der Widerspruch der kapitalistischen Produktionsweise ausgedrückt:

25) So Jörg Huffschmid in: Der Charakter der gegenwärtigen Wirtschaftskrise, in: Blätter tür deutsche und internationale Politik, Nr. 4 (1975), S. 391 
„Die wahre Schranke der kapitalistischen Produktion ist das Kapital selbst, ist dies: daß das Kapital und seine Selbstverwertung als Ausgangspunkt und Endpunkt, als Motiv und Zweck der Produktion erscheint; daß die Produktion nur Produktion für das Kapital ist und nicht umgekehrt die Produktionsmittel bloße Mittel für eine sich stets erweiternde Gestaltung des Lebensprozesses für die Gesellschaft der Produzenten sind." (26)

Das Mittel, die Entfaltung der gesellschaftlichen Produktivkräfte, die Anhäufung von produktivem Kapital, gerät so in ,fortwährenden Konflikt mit dem beschränkten Zweck, der Verwertung des vorhandenen Kapitals." (27) Nicht die beschränkte Nachfrage ist so Ursache der kapitalistischen Krise, sondern der Zweck der Produktion, die Verwertung des vorgeschossenen Kapitals! Dies schlieft allerdings ein: die Entwicklung der Produktîvkräfte über die (zahlungsfähige) gesellschaftliche Nachfrage hinaus, die Nichtabsetzbarkeit von produzierten Waren aufgrund der sinkenden Profitraten und der relativ begrenzten Konsumtion der Massen. Überakkumulation von Kapital ist in diesem Sinne auch die Ursache für die Entwicklung der Arbeitslosigkeit, die das Brachliegen, die Entwertung und Vernichtung von Kapital in wertmäßiger und stofflicher Form begleitet.

Zwar ist es in jedem Zyklus ein und derselbe Prozeß, der ein Zuviel an Kapitalwert im Verhältnis zur vergangenen und erwarteten Profitrate und der zugleich einen Überfluß an produktivem Kapital im Verhältnis zur Nachfrage nach Produkten der beiden Abteilungen sowie eine zyklische Arbeitslosigkeit hervorbringt. Aber die Bedingungen der Produktion von Profit verändern sich von Zyklus zu Zyklus mit der Anhäufung von Kapitalwert und produktivem Kapital in einer langfristigen Prosperitätsphase, wie in Westdeutschland in den 50er und 60er Jahren. Die zyklischen Krisen hatten daher keineswegs eine Entwertung und Vernichtung von Kapital in einem Ausmaß zur Folge, daß Strukturveränderungen in den Akkumulationsbedingungen (wie die Veränderung der organischen Zusammensetzung und der Mehrwertrate) hätten vermieden werden können. Mit der langfristigen Anhäufung von produktivem Kapital wird daher die Notwendigkeit der Entwertung und Vernichtung von Kapital (sowie eine Umstrukturierung der Industriezweige) von Zyklus zu Zyklus dringender. Zugleich drückt sich darin die Steigerung der Produktivkraft der Arbeit aus, die die Nachfrage nach Arbeit relativ züm eingesetzten Kapital abnehmen läßt. Wird - aufgrund der fallenden Verwertung - nicht mehr genügend Zusatzkapital angelegt, so können die freigesetzten Arbeiter nicht mehr vom Produktionsprozeß absorbiert werden, wie sich dies im zyklischen Abschwung seit 1971 herausstellt. Auf diesem Hintergrund werden wir nun versuchen, die langfristigen und die zyklischen Ursachen der Herausbildung einer industriellen Reservearmee in Westdeutschland darzustellen.

26) Kapital III, a.a.O., S. 260

27) ebda. 
Wie aus dem Schaubild ersichtlich, ist die Herausbildung einer industriellen Reservearmee nicht nur Ergebnis der zyklischen Krise, sondern bildet sich spätestens 1971 heraus. Dabei fällt das gegenüber dem früheren Verlauf der Kurve langzeitige Abfallen der Beschäftigung auf: Seit Ende 1971 sinken die Beschäftigungszahlen parallel zu den stagnierenden Investitionen, selbst die Wiederbelebung der Nachfrage $72 / 73$ vermochte nicht das Absinken der Beschäftigung zu vermindern.

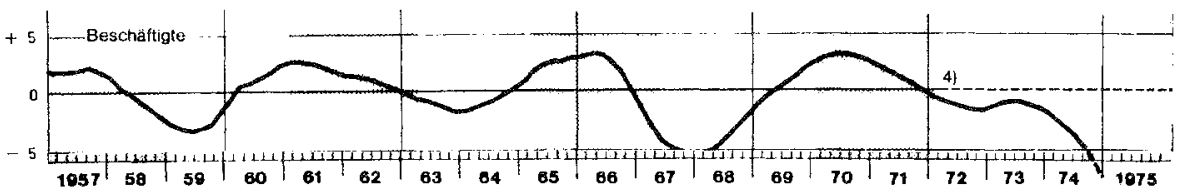

Schaubild 4: Veränderungsraten der Beschäftigten der Industrie

Quelle: $\quad$ Monatsberichte der Deutschen Bundesbank Nr. 7 (1975)

a) Als wichtigste Ursache dieser Entwicklung sind hier die oben dargestellten Strukturveränderungen im Reproduktionsprozeß 3 des Kapitals zu nennen. Es macht sich nämlich in den 70er Jahren die Tatsache geltend, daß die Steigerung der Arbeitsproduktivität und der Mehrwertrate im Laufe der 60er Jahre mehr und mehr nur noch mit einem hohen Kapitalaufwand pro Arbeitskraft, d. h. mit einer hohen technischen Zusammensetzung des Kapitals möglich war. So war im Vergleich zum Jahr 1950 von den einzelnen Kapitalisten durchschnittlich der zweieinhalbfache Kapitalaufwand notwendig, um einen Arbeitsplatz mit Maschinen, Bauten etc. auszurüsten. Oder es läßt sich auch umgekehrt sagen, daß zur Erweiterung der Produktion und des Kapitals im Laufe der 60er Jahre immer weniger Arbeitskräfte nötig waren. Diese Tendenz spiegelt sich auch darin wider, daß der Anteil der direkt dem Kapital subsumierten produktiven Arbeiter gegenïber den in der Zirkulation und im Staatssektor beschäftigten unproduktiven Arbeitem sturrdig abnahm. Gerade wenn - wie vorausgesetzt werden kann - die Profitrate fällt, sinkt aber auch die Kapitalisierung von Profit, wodurch aufgrund des notwendigen hohen Kapitalaufwands pro Arbeitsplatz wieder weniger Arbeiter in den Produktionsprozeß eingegliedert werden können, als (vgl. dazu unten) freigesetzt wurden. (28)

Andererseits geht die Veränderung in den Reproduktionsbedingungen des Kapitals einher mit Konzentrations- und Zentralisationsprozessen. Kleinere Kapitale, die zumeist einen höheren Anteil von Arbeitern ,pro Kapitaleinheit" aufweisen als große und die noch in den 50er und 60er Jahren in der Konkurrenz noch bestehen konnten, unterliegen zunehmend der Konkurrenz der großen Kapitale, 
die hohe Profitmassen auf sich vereinigen können und so den Fall der Profitrate kompensieren können. Dies hat nicht nur eine überproportionale Freisetzung von Arbeitskräften zur Folge, sondern auch eine Verminderung von Beschäftigungsmöglichkeiten überhaupt bei einem emeuten Aufschwung. Wenn kleinere Kapitale aus Konkurrenzgründen das notwendige Kapitalminimum nicht (oder nicht mehr) aufbringen können, fallen sie als Beschäftigungsmöglichkeit aus und die verbliebenen arbeitsintensiven Klein- und Mittelbetriebe absorbieren in einem Aufschwung nurmehr noch eine geringe Anzahl von Arbeitern.

b) Werden schon durch diese Strukturveränderungen die Beschäftigungsmöglichkeiten für produktive Lohnarbeiter eingeengt, so kommt für die 70er Jahre noch ein verschärfender Umstand hinzu: Da die Kapitale mit dem Investitionsboom Ende der 60er Jahre große Massen produktiven Kapitals angehäuft hatten, die ab Anfang der 70er Jahre zusammen mit den steigenden Lohnkosten auf die Profitraten drücken, sind die Kapitale allesamt gezwungen zu rationalisieren, ohne zusätzliche Erweiterungen vorzunehmen. Wie oben gezeigt, herfsehen seit 1971 in den Unternehmen Rationalisienungsinvestitionen (29) vor: neue arbeitssparende Produktionsmethoden werden eingeführt, Arbeiter in der Produktion und auch in der Zirkulation werden freigesetzt. Selbst um die Jahreswende 1972/73 erfolgen - trotz ansteigender Nachfrage und Auslastung der Kapazitâten - kaum Erweiterungsinvestitionęn. Die vorhandenen Kapazitäten wurden voll ausgefahren, von einer Zusatzanlage von Kapital wurde aber aufgrund der profitmindernden Wirkung abgesehen. Statt dessen wurde brachliegendes Geldkapital zur produktiven Anlage ins Ausland transferiert, wie aus Schaubild 5 deutlich wird (dies ist auch die langfristige Tendenz in den 70er Jahren). Oder aber das Geldkapital floß auf Geldmärkte wie den Eurogeldmarkt zum Zwecke der Spekulation.

29) Zum Charakter der Investitionen als „Rationalisierungsinvestitionen “ vgl. Sachverständigenrat JG 1971; Ziff. 32; JG 1972, Ziff. 141; JG 1973, 68: „Bemerkenswert viele Unternehmen planten (. . .) mit ihren Investitionen neue Produktionsmethoden einzuführen. Das Bemühen um technischen Fortschritt, das sich darin äußert, war sonst nur im Abschwung so groß." (Angesprochen ist hier der Wiederaufschwung der Nachfrage um die Jahreswende 1972/73) JG 1974, Ziff. 88. Dabei steigt der Anteil der bloßen Ersatzinvestitionen, die bei der vorherrschenden Technik auch einen Freisetzungseffekt haben, an. 


\section{Dextsche Direktinvestitionen in Ausland - In Millinden DM}

\section{So wuchsen die Auslands- \\ Diese Branchèn investierten \\ Investitionen:}

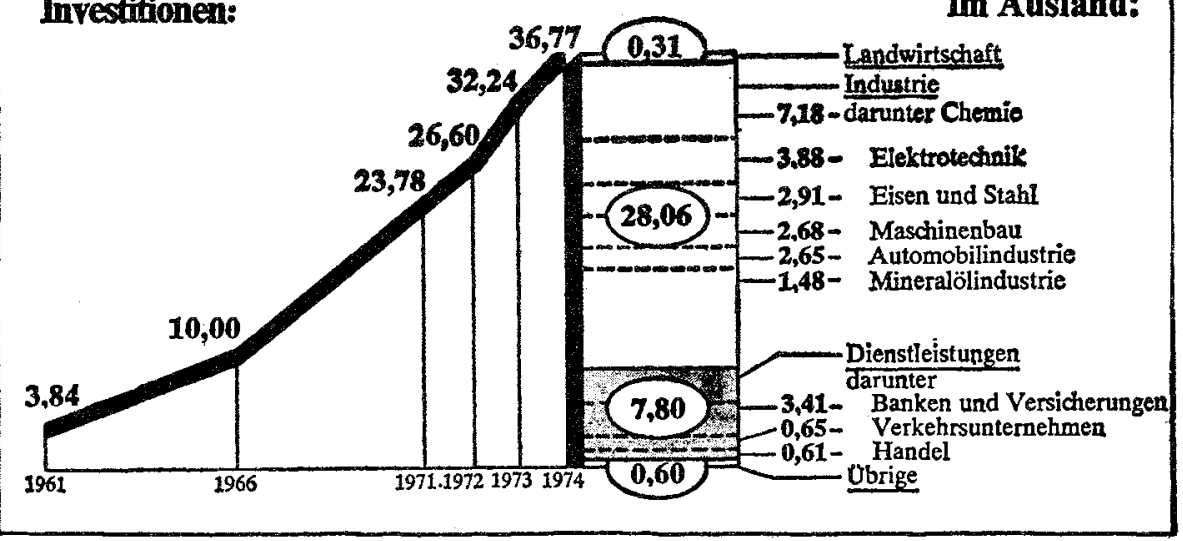

Schaubild 5: Deutsche Direktinvestitionen im Ausland 1961 bis 1974

Quelle:

Handelsblatt vom 18./19.7.1975

Daher können die aufgrund der oben gezeigten strukturellen Veränderungen freigesetzten Arbeiter schon in Phasen scheinbarer Prosperität und stagnierenden oder sogar noch kurzfristig ansteigenden Investitionen nicht mehr in den kapitalistischen Produktionsprozeß eingegliedert werden.

Die Ursachen für die Herausbildung einer industriellen Reservearmee sind daher zwar durchaus langfristiger Natur (steigende technische Zusammensetzung des Kapitals, Kapitalkonzentration und arbeitssparende Innovationen und Rationalisierungen); diese Trends machen sich aber gerade im zyklischen Abschwung stark geltend, wenn das angehäufte produktive Kapital nicht mehr ausgelastet werden kann. Während nämlich die Strukturveränderungen die Beschäftigungsmöglichkeiten einengen, erfolgen direkt Freisetzungen von Arbeitskräften, wenn die Akkumulation nachläßt, die Erweiterungen von Anlagen zurückgehen und Rationalisierungsinvestitionen vorherrschen.

Solange überdies die Unternehmen ihr produktives Kapital noch voll auslasten können, ist auch der Prozefs der Brachlegung und Vernichtung kleinerer Kapitale noch nicht sichtbar. Aber bereits bei den ersten Anzeichen einer nachlassenden Nachfrage - etwa in bestimmten Bereichen - macht sich der Druck der großen Kapitale gegenüber den kleineren geltend. So gab es 1973/74 die meisten Pleiten in der Geschichte Westdeutschlands. (30) Demgegenüber können sich jene Kapi-

30) Vgl. FR vom 24.12.1974: 1973 gab es 5 515, 1974 bereits 7800 Pleiten. Das von den Konkursen zunächst wesentlich die kleinen Kapitale betroffen waren, zeigt J. Huff- 
tale und Branchen noch bis in die Krise hinein im Konkurrenzkampf halten, die aufgrund hoher Arbeitsproduktivitäten, noch vorhandener Nachfrage, der Möglichkeit, gestiegene Kosten auf die Preise überzuwälzen (um so Entwertungsprozessen zu entgehen (31)) imstande sind, profitabel zu produzieren. Bei der Analyse der gegenwärtigen Arbeitslosigkeit sind daher noch die branchenspezifischen Strukturveränderungen zu betrachten, die allerdings nur auf dem Hintergrund der allgemeinen Entwicklung des Kapitals ihr besonderes Gewicht bekommen.

c) Gerade jene Branchen gerieten in den letzten Jahren mehr und mehr in Schwierigkeiten, die die oben genannten Kompensationsmöglichkeiten nicht mehr aufwiesen.

Wir müssen hier in Westdeutschland zwei Gruppen von Branchen unterscheiden, die besonders stark von Krisentendenzen betroffen waren und es noch sind, und deren Besonderheit hier innerhalb der allgemeinen Analyse kurz dargestllt werden soll:

(1) Im Zuge der langen Prosperitätsphase in der BRD haben sich gegenüber der langfristigen gesellschaftlichen Nachfrage in den Branchen der Fahrzeugbauund dek Bauindustrie große Überkapazitäten von produktivem Kapital herausgebildet, die jetzt in einer schweren "Strukturkrise“ abgebaut werden: die Gesellschaftlichkeit der Produktion macht sich in der Entwertung, Brachlegung und Vernichtung von Kapital und in der Freisetzung von Arbeitern hier besonders einschneidend geltend. In der Bauindustrie ist dies neben der Sättigung des Wohnungsmarkts und der Bauspekulation, die jetzt zusammengebrochen ist, auch auf das Zurückstellen von Erweiterungsinvestitionen der Kapitalisten zurückzuführen. Die Fahrzeugindustrie trifft nicht nur die relative Sättigung des inländischen Marktes und die Verteuerung des Benzins, sondern auch die Verschlechterung der Exportmöglichkeiten durch die veränderten

schmid a.a.O., S. 402. Wir können allerdings seinen hierauf basierenden Thesen zur „Polarisierung zwischen monopolistisctien und nichtmonopolistischen Kapitalen " nicht folgen: H.'s Tabelle würde im Jahr 1975, in dem auch die eher monopolistischen Unternehmen der Wachstumsindustrien (Stahl, Chemie, Maschinenbau, Elektrotechnik ...) in die Krise geraten (mit entsprechenden Freisetzungsraten!) andess aussehen, zumal die in Bedrängnis geratenen Kleinkapitale bestimmt nicht die Hand des antimonopolistischen Bündnisangebots ergreifen, sondern sich gerade an der Ware Arbeitskraft schadlos halten werden! Was H. unter Entwertung faßt, reduziert sich übrigens bei genauem Hinschauen auf „Vernichtung von Kapital“.

31) So setzt der Sachverständigenrat in die aktuelle Krise die Hoffnung, daß nunnehr auch die Unternehmen, ,deren Rentabilität bislang wegen der fortschreitenden Inflation noch gesichert schien“, veranlaßt werden, „verstärkt Produktionsreserven aufzuspüren und ihre Investitionen dorthin (...) verlagern, wo eine produktivere Nutzung der volkswirtschaftlichen Ressourcen möglich ist." (JG 1974, Ziff. 224). Dies scheint 1975 besonders auf die Grundstoff- und Produktionsgüterindustrie zuzutreffen, die von Ausbleiben der ausländischen Nachfrage jetzt getroffen werden, nachdem sie zuvor Entwertungsprozessen sich noch entziehen konnten. Die mronetäre Akkumulation kann hier die reale nicht mehr vermitteln. 
Wechselkurse und gestiegene Transportkosten nach der Ölkrise. Allerdings: hier we oben gilt natülich Nachfrage nur, soweit sie als zahlungsfähige Nachfrage auftritt.

(2) Abgeschirmt durch relatix ginstige Wechselkurse konnte sich auch die Textil:; Bekleidungs und Lederindustrie während der langen Prosperitätsphase in Westdeutschland noch gut entwickeln. Dabei stand sie in scharfer Konkurrenz zu sogen. Niedriglohnlandern des Mittelmeerraumes und der „Dritten Welt". Die Nachteile eines relativ höheren Lohns (32) konnten allerdings noch durch eine hohe Arbeitsproduktivität und günstige Wechselkurse aufgehoben werden. Mit dem Wegfall der günstigen Wechselkurse aber konnten die niedrigen Löhne der Konkurrenz nicht mehr durch eine Erhöhung der Arbeitsproduktivität ausgeglichen werden, da dies unter den Bedingungen dieser Branche eine beschleunigte Anlage von Kapital erfordert hätte. Zugleich fiel aufgrund der verschärften Konkurrenz auf dem Weltmarkt die Möglichkeit aus, die erhöhten Kosten auf die Preise überzuwälzen: Die Branche geriet als erste in die Krise, hatte mit die höchsten Konkursżahlen und Freisetzungen (relativ zur beschäftigten Arbeiteranzah1). (33)

d) Die frihzeitig eingetretene Krise und die Massenarbeitslosigkeit in der Automobil-, Bau, Text1- und Bekleidungsindustrie war allerdings nu r ein Vorbote der eigentlicher zuklischen Krise und zyklischen Überakkumulation. Indem als Folge der sich verschärfenden Überakkumulations- und Verwertungsprobleme des Kapitals sowohl die Konsum- als auch die Investitionsnachfrage schnell zurückging, bahnte sich bereits eine zyklische Massenarbeitslosigkeit an, deren Ausmaße alle bisherigen in der Geschichte der BRD übertrifft und die sich allmählich auf eine dritte Gruppe von Branchen verschiebt, die der Irvestitionsund Grutindstoff : and Produktionsgüterindustrien. Denn nicht nur die Konsumgïterindustrie (hier insbes. die langlebigen Konsumgüter), sondern auch die Investitionsgüterindustrie (z. B. der Maschinenbau) wurde betroffen; die Krise brach selbst in der Grundstoff- und Produktionsguterindustrie zu Beginn des

32) Dabei ist zu beachten, daß die Arbeiter in den benannten Branchen am Ende der Lohnskala in der Bundesrepublik stehen. Bedenkt man, daß die Löhne z. B. in Hongkong, das mit den Branchen in der BRD konkurriert, $51 / 2$ mal niedriger sind, wird deutlich, welche schlechte Weltmarktposition diese Branchen haben. (Vgl. zur Lohnskala: Osterland u.a., Materialien zur Lebens- und Arbeitssituation der Ind ustriearbeiter in der BRD, Ffm 1973, Tab. 92 im Anhang; zum Lohnkostenvergleich auf dem Weltmarkt: P.G. Rogge, Geänderte Standortqualitäten der Bundesrepublik Deutschland im internationalen Wettbewerb, vervielfältigtes Manuskript, o.0., 1974)

33) Vgl. Monatsberichte der Deutschen Bundesbank, Statistische Beihefte, Reihe 4, April 1975, S. 8: Während die anderen Branchen im Frühjahr 1973 noch Zuwachsraten aufweisen, sinkt das Produktionsvolumen zuerst in der Textil- und dann in der Fahrzeugindustrie bereits ab! Zum allgemeinen Zusammenhang von Kapitalintensität, Arbeitsproduktivität und Lohnhöhe auf dem Weltmarkt vgl. genauer Wolfgang Schoeller, Die Bedeutung des Weltmarktzusammenhangs zwischen ökonomisch unterschiedlich entwickelten Ländern für den kapitalistischen Wertbildungsprozeß, Diss. FU Berlin 1975 
Jahres 1975 beschleunigt aus. Daher hatten im Verlaufe dér Krise auch die Eisenund Stahlindustrie und die Chemieindustrie - die noch lange günstige Exportund Absatzsphären aufwiesen - Absatzschwierigkeiten, Produktionseinschränkungen und Entlassungen zu verzeichnen. Die Tiefe der zyklischen Krise und der gleichzeitige Weltmarktabschwung - der 1975 erst voll einsetzte - mußten daher auch die der Investitionsgüterindustrie ,vorgelagerten“ Branchen ergreifen, die lange Zeit hindurch noch keine Krisenanzeichen aufwiesen. Während bereits andere Branchen der Konsum- und Investitionsgiterindustrie und auch die Automobil-, Bau- und Textilindustrie infolge von Kapazitätsabbau, Kapitalvernichtung und -entwertung ihren „konjunkturellen Tiefpunkt", weitgehend erreicht haben, geht die Krise in den zuletzt genannten Branchen noch weiter.

So treffen in der Krise 1974/75 als Ursachen der Arbeitslosigkeit dielangfristigen Strukturveränderungen in der Kapitalzusammensetzung, die Kapitalkonzentration, die Veränderung der Industriestruktur (Überexpansion der Abteilung I) zusammen mit der zyklischen Massenarbeitslosigkeit. Daher ist die Arbeitslosigkeit aueh nicht über den zyklisehen Aufschwung der Produktion allein zu beheben. Diese Vorstellung einer möglichen Beseitigung der Arbeitslosigkeit im Aufschwung liegt den Konjunkturprogrammen der Bundesregierung zugrunde, deren Grenzen wir jetzt aufzeigen können.

4. STAATLICHES KONJUNKTURPROGRAMM GEGEN ARBEITSLOSIGKEIT?

Mit dieser krisenhaften Entwicklung 1974 war zwar die Bundesregierung frühzeitig von seiten der Gewerkschaften aufgefordert worden, die im Stabilitäts- und Wachstumsgesetz vorgesehenen Instrumente der Globalsteuerung gegen die Krise und Arbeitslosigkeit einzusetzen. Dennoch kam es zunächst nicht zu einem solchen Eingreifen. Wie ist dieser "time-lag" zwischen dem Konjunktureinbruch und dem erst Ende 1974 beschlossenen Konjunkturprogramm der immerhin von der Sozialdemokratie weitgehend bestimmten Regierung zu erklären? Um nicht in den Fehler zu verfallen, hier wären wieder einmal die falschen Männer am Ruder gewesen (wie die Gewerkschaften sie im Direktorium der Bundesbank auszumachen glaubten), muß auf eine Reihe ökonomischer und sozialer Zwänge hingewiesen werden, denen die Wirtschaftspolitik der Bundesregierung ausgesetzt war, und die noch einmal die Bedeu tung der Krise für den kapitalistischen Wachstumsprozeß herausheben.

Die ab Ende der 60er Jahre und Anfang der 70er Jahre sprunghaft angestiegenen Preissteigerungsraten waren die Grundlage dafür, daß die Fiskal- und Geldpolitik vorwiegend von (versuchten) Restriktionsmaßnahmen beherrscht war. Insbesondere die Bundesbank und Kapitalverbande drängten auf Antiinflationsmaßnahmen, die sich auch in einem Antiinflationsprogramm 1973 niederschlugen. (34)

34) Das Stabilitätsprogramm umfaßte im wesentlichen den „Stabilitätszuschlag“, eine Investitionssteuer, die Aufhebung der degressiven Abschreibungen und der Sonderabschreibungen nach $\S 7 \mathrm{~b}$ (ESTG) 
(Diese Maßnahmen mußten allerdings bereits Ende 1973 wieder aufgehoben werden). Gerade die großen Kapitale hatten insofern noch ein Interesse an einem Restriktionsprogramm, als sie sich davon:

- durch niedrige Preissteigerungsraten günstige Konkurrenzbedingungen auf dem Weltmarkt erhofften (nicht zu Unrecht, wie sich herausstellte), und

- einen Druck auf gewerkschaftliche Geldlohnforderungen und gesellschaftspolitische Programme versprachen.

Denn der vorangegangene Boom hatte ja - wie schon dargestellt - nicht nur die nach der Krise 1966/67 günstigen Verwertungsbedingungen erodieren lassen, sondern auch die betriebliche und gesellschaftliche Stellung der Arbeiter und Gewerkschaften gestärkt, was auch in entsprechenden Lohnforderungen seinen Ausdruck fand. Da also die soziale Machtstellung der Arbeiter und Gewerkschaften in Prosperitätsphasen ansteigt, könnten eine restriktive Fiskal- und Geldpolitik und eine ,gewisse Rate der Arbeitslosigkeit" als Mittel eingesetzt werden, um die ökonomischen und sozialen Erfolge der Arbeiter zu bremsen. (35) Zumal sich die großen Kapitale weitgehend in dieser Phase noch über das Ausland refinanzieren konnten und so den eingeschlagenen Restriktionskurs unterliefen.

Aber selbst als die Krise 1974 immer stärker hervortrat, blieben Gegensteuerungsmaßnahmen zu nächst noch aus. Dies wurde auch begründet: Einmal sprach die Bundesregierung offen von notwendigen Anpassungsprozessen, die man nicht aufhalten wollte um des künftigen Wachstums willen. Hier wurde also ganz offen ein in der Krise sich durchsetzender Strukturwandel gefördert: Kapitalentwertung und Kapitalvernichtung und Freisetzungen in bestimmten Branchen (angesprochen waren die Bau-, Fahrzeugbau- und Textilindustrie) wurden hingenommen, Jediglich soziale Flankierungsmaßnahmen angeboten. Zum anderen stiegen - entgegen der ökonomischen Lehrbuchweisheit, die auch dem Stabilitäts- und Wachstumsgesetz zugrunde lag - die Preise auch in der beginnenden Krise weiter, wenn auch leicht abgeschwächt. Ein frühzeitiges Durchstarten - so meinte man - hätte einen zu hohen Inflationssockel im nächsten Boom zur Folge gehabt. Drittens aber hielten Bundesbank, Kapitalverbände und die bürgerliche Öffentlichkeit die Regierung dazu an, mit möglichen Konjunkturprogrammen bis zu den Ende 1974 fälligen Tarifverhandlungen $\mathrm{zu}$ warten, die erst die Voraussetzungen für einen neuen Aufschwung durch niedrige Lohnsteigerungen (und - so wurde gefordert sinkende Reallöhne) schaffen sollten. (36) Erst nachdem die Tarifabschlüsse deutlich unter den gewerkschaftlichen Forderungen (und selbst unter der von der Bundesregierung vorgeschlagenen Obergrenze) lagen, legte die Regierung ein Konjunkturprogramm vor, als abzusehen war, daß nicht nur $5 \%$ Inflationsrate, sondern auch $5 \%$ Arbeitslosenquote auf die westdeutsche Wirtschaft zukamen.

35) Wir meinen allerdings, daß dem eher ein Aufgreifen vorhandener Tendenzen - sei es der Geld- und Kreditkrise als Folge des beginnenden Abschwungs, sei es der sich herausbildenden Akkumulationskrise mit der sie begleitenden Arbeitslosigkeit - und nicht eine autonome politische Aktion zugrunde liegt!

36) Vgl. etwa die Forderung des RWI nach $+4 \%$ Lohnerhöhung 1975, was einer Reallohnsenkung um über $3 \%$ gleichkommt. FR vom 14.2.1975 
(a) Worauf zielten die im Dezember 1974 eingeleiteten Maßnahmen? (37)

- Zunächst wesentlich auf die Förderung der privaten Investitionen, indem auf den Angelpunkt der „ups and downs“ des industriellen Zyklus' und das Kardinalproblem der gegenwärtigen Überakkumulationskrise, die Profitrate, eingewirkt wurde. Durch eine 7,5 prozentige Investitionszulage sollte der akkumulierte Profit (und damit die Investition) erhöht werden. Dem durch Steuermindereinnahmen und Steuerreform geschmälerten Bundeshaushalt soll diese Maßnahme allein $19768 \mathrm{Mrd}$. DM kosten.

- Daneben sollte durch die Steuerbegünstigung nach dem $\S 7$ b des Einkommensteuergesetzes, der auf den Zweiterwerb von Häusern und Wohnungen ausgedehnt wurde, die Nachfrage im Bausektor erhöht werden. Zugleich wurden staatliche Investitionsprogramme, die ebenfalls die Nachfrage im Bausektor, aber besonders den Energiesektor fördern sollten (langfristige Sicherung von Energie als allgemeiner Produktionsbedingung), beschlossen.

- Auf die Stärkung der Massenkaufkraft und Hebung der Konsumnachfrage zielten die Steuer- und Kindergeldreform (14 Mrd. DM Zuwachs der Massenkaufkraft). Allerdings waren diese Maßnahmen eher davon bestimmt, die enormen Ungerechtigkeiten in dér Steuerbelastung (bes. bei der Lohnsteuer) zu beseitigen.

Zusätzliche staatliche Ausgaben zur Steigerung der Masseneinkommen und damit der Konsumnachfrage, Subventionen im Solzialbereich zur Steigerung der Einkcmmen der unteren Einkommensschichten waren - obwohl durchaus traditionelle Mittel einer keynesianischen Antikrisenpolitik gerade unter sozialdemokratischen Regierungen - nicht vorgesehen.

- Dagegen beeilte man sich, den Unternehmern die Last der Lohnkosten durch Lohnkostenzuschüsse für neugeschaffene Arbeitsplätze zu nehmen. Um Disproportionalitäten und die „Immobilität der Arbeitskräfte" auf dem Arbeitsmarkt $\mathrm{zu}$ beheben, wurden außerdem Mobilitätszulagen für Arbeiter vorgesehen, die ihre Wiedereingliederung in anderen Branchen und Regionen fördern sollten.

Obwohl dieses Programm exakt die Krisensituation in der BRD ausdrückt, ist es doch in mehrerer Hinsicht erstaunlich. Es zeigt einmal, wie schwer die strukturellen Aspekte in der Kapitalakkumulation (Überakkumulation- stagnierende Kapitalakkumulation) sein müssen. Maßnahmen, die fast ausschließlich auf die Subventionierung der Investitionen und auf die Profitabilität des Kapitals zielen, sind selbst in der Geschichte des Keynesianismus selten. Dies kann nur als Indiz dafür gelten, daß die Profitabilität des Kapitals sehr schlecht ist und massive Begünstigungen notwendig sind, um die Akkumulation wieder zu beschleunigen. Zugleich ist das durchgefuhrte Programm aber auch ein Indiz für die Schwäche der Gewerkschaften und der Linken in der BRD, die nicht in der Lage waren, ein anderes Konjunkturprogramm - wie es z. B. in anderen westeuropäischen Ländern erzwungen werden konnte -- 
durchzudrücken. Gerade die fast fehlenden staatlichen Wohlfahrtsausgaben, die fehlende Subventionienung des Massenkonsums und des Einkommens der unteren Schichten, machen deutlich, wie schwach selbst sozialreformerische Kräfte innerhalb und außerhalb der Regienng sind. Selbst auf - immer notwendiger gewordene - Infrastrukturausgaben im Bildungswesen, im Verkehrswesen, im Umweltbereich und Sozialwesen wurde verzichtet. Nur der Energiebereich wurde gefördert - ein Bereich, der direkt die Konkurrenzfahigkeit des deutschen Kapitals auf dem Weltmarkt betrifft. Auch dann wird wieder die allgemeine Zielrichtung des Programms deu tlich.

(b) Kann das Konjunkturprogramm die Vollbeschä ftigung wieder herbeifuhren?

Das rechtskeynesianische Programm wird nun aber von der SPD gerade mit der Begründung verteidigt, nur über die Förderung der Investitionen könnten die Arbeitsplätze erhalten bleiben: „Die Investitionen von heute schaffen die Arbeitsplätze und Einkommen von morgen." (38) Darin drückt sich natürlich auch - unter den gegebenen gesellschaftlichen Machtverhältnissen - eine. Anerkennung von kapitalistischen Gesetzmäßigkeiten aus, die in naiven Konzepten der Nachfragesteuerung durch Staatsausgaben nicht vorhanden war. Indem in den Investitionen, also letztlich im Profit, der Ansatzpunkt der staatlichen Aktion gesehen wird, wird gesehen, daß das Wachstum der Wirtschaft und der Erhalt der Arbeitsplätze zunächst und zu allererst vom akkumulierten Profit abhängt.

Erinnern wir uns daran, daß sich die Profitrate erst wieder durch die Krise hebt, so ist es nicht von ungefähr, daß das Programm erst zu einem Zeitpunkt eingesetzt hat, $\mathrm{zu}$ dem die Wirkung der Krise bereits in der Entwertung, Vernichtung, Konzentration und Zentralisation von Kapital und der Lohnsenkung spürbar wurde. Wir gingen oben bereits darauf ein. Wenn das lange Zögern nun mit den noch hohen Inflationsraten begründet wurde, so drückte sich darin ja gerade aus, daß ein Aufschieben von Entwertungsprozessen über inflationäre Preisentwicklung nicht mehr toleriert werden konnte. Die staatliche Konjunkturpolitik baute so auf den Wirkungen der Krise auf, ohne jedoch den weiteren Verlauf der Akkumulation entscheidend bestimmen zu können. Dies soll im folgenden an den Grenzen des Konjunkturprogramms demonstriert werden:

- Es ist keineswegs sicher, ob die Wirkungen der Krise bereits einen solchen Kapitalvernichtungsprozeß ingangsetzen konnten, daß tatsächlich ein schneller Wiederaufschwung der Profitabilität von dieser Seite her gesichert ist. Sowohl die immer noch hohen Inflationsraten als auch die weiterhin stagnierende bzw. sinkende Kapitalakkumulation deuten darauf hin, daß die strukturellen Verwertungsprobleme auch in absehbarer Zeit nicht aufgehoben werden, daß vielmehr die Profitrate auf niedrigem Niveau sich bewegen wird. Wir können dies 
an mehreren Indikatoren verdeutlichen, wozu allerdings gesagt werden muß, daß in den verwendeten Prognosen gerade die ,reinigende Wirkung" von Krisen nicht berücksichtigt wird:

(1) wird die Investitionsquote in den 70er Jahren auf niedrigem Niveau verharren,

(2) wird der Schwerpunkt der Investition bei Rationalisierungsinvestitionen liegen und

(3) werden die Kapitale dabei wesentlich Ersatzinvestitionen vornehmen, überschüssige Profitteile dagegen teilweise im Ausland anlegen. (39) Diese Indikatoren deuten also auf eine niedrige Profitrate und mangelnde Zusatzanlage von Kapital in den 70er Jahren hin, was auch schon im Sachverständigen-Gutachten (1974, Ziff. 222) deutlich geworden ist. Bleibt daher die Profitmasse trotz Investitionszulage im Verhältnis zum vorhandenen Kapitalwert so niedrig, daß sich eine Zusatzanlage angesichts der erwarteten Profitrate und des Auslastungsgrads des produktiven Kapitals nicht lohnt, so wird die Investitionszulage kaum gesamtwirtschaftliche Wirkungen zeigen. Eher ist zu vermuten, daß diese Zulage mit dazu dient, Rationalisierungsinvestitionen auf Basis der vorherrschenden arbeitssparenden Technologie, wie sie aufgrund des Drucks der sinkenden Verwertung seit 1971 in Westdeutschland vorherrschen zu finanzieren (vgl. dazu Tabelle 1)'. Solche Investitionen sind ja Reflex auf eine gestiegene Zusammensetzung des Kapitals und gestiegene Lohnkosten: über die Steigerung der Produktivkraft der Arbeit und die Freisetzung von Arbeitskräften sollen die Mehrwertrate erhöht und der Lohnkostenvorschuß verringert werden. Angesichts der auch in absehbarer Zeit vorherrschenden Verwertungsbedingungen dürften daher nicht nur die sowieso sinkenden Zusatzanlagen, sondern besonders die Ersatzanlagen von Kapital solche "Rationalisierungseffekte" haben, während zugleich nicht ausreichend zusätzliche Investitionen im Inland getätigt werden, um die freigesetzten Arbeiter wieder in den Produktionsprozeß rückgliedern zu können. Das heißt dann aber: Die Investitionen von heute schaffen die Arbeitsplätze von morgen . . . ab!

39) Nach dem Ifo-Schnelldienst vom 4.3.1975 stagnieren die Investitionsquoten in der deutschen Industrie 1975-1979 auf niedrigem Niveau, soweit sich dies aus den Investitionsplänen der Industrie ablesen läßt. Vgl. dazu auch Schaubild 1. Schwerpunkt der Investition wird die Rationalisierung sein; während die Zahl der Beschäftigten im Inland nicht ausgeweitet werden soll, werden die Auslandsinvestitionen verstärki werden: „Nach wie vor planen drei Viertel der in Frage kommenden Unternehmen, ihre Investitionen stärker im Ausland als im Inland auszudehnen." (S. 7). Dabei ist - nach einer Umfrage des Allensbacher Instituts für Demoskopie - „die Neigung zu Auslandsinvestitionen von der gegenwärtigen wirtschaftlichen Situation weitgehend unabhängig." (Zit. in der FR vom 8.1.1975). Demnach bleiben die Bedingungen für eine strukturelle Arbeitslosigkeit, wie wir sie unter 3 dargestellt haben, weiterhin erhalten! 
TABELLE I: Zielsetzung der Investition nach Industriegruppen 1975 in \% geplant

Kapazitätserweiterung Rationalisierung Ersatzbeschaffung

$\begin{array}{llll}\text { Grundstoff und Produktions- } & 31 & 41 & 28 \\ \text { güterindustrie } & 27 & 49 & 24 \\ \text { Investitionsgüterindustrie } & 21 & 60 & 19\end{array}$

Quelle: ifo-Schnelldienst vom 12.6.1975; zitiert nach Blätter für deutsche und internationale Politik, Nr. 7/1975, S. 819

Auch von der Nachfrageseite her sind der staatlichen Wirtschaftspolitik auf absehbare Zeit Grenzen gesetzt. Eine Erhöhung der ,effektiven Nachfrage“ durch staatlichen Verbrauch und staatliche Investitionen, die dazu dienen mögen, den Auslastungsgrad des angehäuften produktiven Kapitals zu erhöhen, ist nicht nur von möglichen inflationären Effekten her beschränkt. Daruberhinaus sind auch die Mittel der Finanzierung weitgehend ausgeschöpft! (40) Soll also nicht die Akkumulation des produktiven Kapitals von einer entsprechenden Akkumulation von Staatsschuld und steigender Zinslast für die öffentlichen Haushalte begleitet sein, dann kann auf lange Frist der Anteil des Staats am Nettoprodukt, der ja den Anteil des Kapitals begrenzt, nicht mehr ausgeweitet werden. Denn dies hätte entweder Steuererhöhungen (also auch eine Erhöhung des steuerlichen Abzugs vom Profit) und/oder u.U. inflationäre Prozesse aufgrund der Zirkulation von Staatsschuldtiteln zur Folge.

Die Wirtschaftspolitik kann so einerseits nicht mehr unbegrenzt zur Auslastung des produktiven Kapitals beitragen, die Kasse ist leer, jede weitere Staatsverschuldung läßt politische Konflikte aufbrechen. Allerdings sieht man doch - keynesianisch orientiert - in der mangelnden effektiven Nachfrage den Kern der Krise und die Möglichkeit des Staatseingriffs, so jedenfalls die Gewerkschaften und Teile der Sozialdemokratie.

Auf der anderen Seite können nun diese Grenzen der staatlichen Intervention auch dadurch nicht behoben werden, indem Löhne gekürzt oder Lohnzunahmen durch den Staat begrenzt werden, um die Profitabilität des Kapitals wieder zu heben. Denn will man die Vernichtung von brachliegendem Kapital und Arbeitslosigkeit verhindern, kann auch nicht das Absinken der Konsumnachfrage und damit die weitere Nichtauslastung von produktivem Kapital in Kauf genommen werden.

40) Es zeigt sich also nicht nur im Scheitern der „Inneren Reformen“, sondern schon bei Analyse der antizyklischen Wirtschaftspolitik, daß hier der Staat über das Geld an den Reproduktions- und Akkumulationsprozeß des Kapitals angebunden ist und keineswegs als autonome Instanz ,steuern" kann. Zur wachsenden Staatsschuld, der Minister Friedrichs in hergebrachter Weise mit der Kürzung der Sozialausgaben begegnen will, vgl. Apel in einem Interview mit der FR (17.12.74): „Wir haben kein Geld!“. Nach Handelsblatt vom 21.11.1974 wird der ,offentliche Schuldenberg“" von 126 Mrd. DM (1970) auf 245 Mrd. DM (1975) angewachsen sein. 
So kommt es, daß die „Verbraucher" aufgefordert werden, weniger zu sparen und mehr zu konsumieren (Schmidt), während zur gleichen Zeit der Wirtschaftsminister Friedrichs - um die Profitabilität der Kapitale bemüht - die Kürzung der Sozialhaushalte, der "Soziallasten“ der Kapitale und des Konsumzuwachses anvisiert. (41) Beide drücken je eine Seite der kapitalistischen Überakkumulationskrise aus, Friedrichs und Schmidt stehen in dieser Weise für das Dilemma staatlicher Wirtschaftspolitik in der Krise.

Diesem Dilemma versucht man allerdings auch dadurch $\mathrm{zu}$ entfliehen, indem die ökonomische und politische Machtstellung des westdeutschen Kapitals auf dem Weltmarkt von der sozialliberalen Regierung bewußt ausgespielt wird. Stabilitätsprogramm, Konzertierte Aktion, Finanzierung der Auslandsmärkte (Italien!), Verstärkung des politischen Einflusses in der EG usw. weisen auf den Versuch der Regierung hin, eine Externalisierung der Krisentendenzen durch Niedrighalten von Inflationsraten und Aufrechterhalten einer hohen Exportquote einzuleiten.

Fassen wir zusammen: Insgesamt sind nicht nur dem staatlichen Eingreifen Grenzen gesetzt, die dem kapitalistischen Akkumulationsprozeß immanent sind. Es wird vielmehr auch deutlich, daß selbst eine Wiederbelebung der Investitionstätigkeit und damit ein Wiederaufschwung der Akkumulation von einem hohen „Sockel“" von Arbeitslosigkeit begleitet werden wird. Denn

1. werden Rationalisienungs- und Ersatzinvestitionen auf der Grundlage arbeitssparender Technologie weiterhin in den 70er Jahren vorherrschen;

2. werden die Kapitale verstärkt im Ausland überschüssige Profitteile anlegen, während die Anlagen im Inland nur noch beschränkt ausgedehnt werden;

3. werden arbeitsintensive Branchen stark ,,schrumpfen “;

4. fördert die sinkende Profitabilität Konzentrations- und Zentralisationsprozesse, die wiederum von der ,Freisetzung" von Arbeitern begleitet sind;

5. bleibt die Überexpansion der Abteilung I und deren Weltmarktabhängigkeit als zentrales Problem.

Dabei ist die zentrale Bedingung für die Herausbildung einer industriellen Reservearmee die im Verhältnis zur ,freigesetzten Arbeitsmasse" zu geringe (und „arbeitssparende“) Zusatzanlage von Kapital aufgnund der Verwertungsbedingungen im Inland!

Eine manifeste industrielle Reservearmee wird daher nicht mehr Ausnahmeerscheinung sein, sondern auch in der BRD zur kapitalistischen Normalität gehören, mit der die Arbeiterklasse und die Gewerkschaften konfrontiert werden. Sie wird Bedingung für die Aufrechterhaltung des Kapitalverhältnisses. Auch ein weiteres Konjunkturprogramm (im Herbst 1975) wird an diesen ökonomischen Zusammenhängen und Problemen nicht viel rütteln können.

41) Nach dem Tagesspiegel vom 30.5.1975 rief Bundeskanzler Schmidt die Bevölkerung zu größerem Konsum auf: Mehr Konsum sei „für den Aufwärtstrend nur förderlich“. Anders Friedrichs, mehr auf die längere Frist und die Investitionstätigkeit bedacht, am 2.6.1975 in der Frankfurter Rundschau: ,Wenn Beschäftigung und Arbeitsplätze auch im kommenden Jahrzehnt gesichtert werden sollten, werde das nur über höhere private und staatliche Investitionen und einer damit verbundenen Einschränkung des Konsumzuwachses gelingen." 